\title{
ZOMG III: The effect of Halo Assembly on the Satellite Population
}

\author{
Enrico Garaldi, ${ }^{\star} \dagger$ Emilio Romano-Díaz, Mikolaj Borzyszkowski and Cristiano Porciani \\ Argelander Institut für Astronomie, Auf dem Hügel 71, Bonn, D-53121, Germany
}

Accepted XXX. Received YYY; in original form ZZZ

\begin{abstract}
We use zoom hydrodynamical simulations to investigate the properties of satellites within galaxy-sized dark-matter haloes with different assembly histories. We consider two classes of haloes at redshift $z=0$ : 'stalled' haloes that assembled at $z>1$ and 'accreting' ones that are still forming nowadays. Previously, we showed that the stalled haloes are embedded within thick filaments of the cosmic web while the accreting ones lie where multiple thin filaments converge. We find that satellites in the two classes have both similar and different properties. Their mass spectra, radial count profiles, baryonic and stellar content, and the amount of material they shed are indistinguishable. However, the mass fraction locked in satellites is substantially larger for the accreting haloes as they experience more mergers at late times. The largest difference is found in the satellite kinematics. Substructures fall towards the accreting haloes along quasi-radial trajectories whereas an important tangential velocity component is developed, before accretion, while orbiting the filament that surrounds the stalled haloes. Thus, the velocity anisotropy parameter of the satellites $(\beta)$ is positive for the accreting haloes and negative for the stalled ones. This signature enables us to tentatively categorize the Milky Way halo as stalled based on a recent measurement of $\beta$. Half of our haloes contain clusters of satellites with aligned orbital angular momenta corresponding to flattened structures in space. These features are not driven by baryonic physics and are only found in haloes hosting grand-design spiral galaxies, independently of their assembly history.
\end{abstract}

Key words: galaxies: formation - galaxies: structure - galaxies: haloes - galaxies: evolution - Local Group

\section{INTRODUCTION}

The standard model of cosmology is based on conventional physics and assumes that the dominant terms of the stress-energy tensor are a cosmological constant and cold dark matter. In this $\Lambda \mathrm{CDM}$ scenario, self-gravitating structures form hierarchically through mergers of smaller units. The end product of this process is a collection of dark matter haloes containing a large amount of substructures that are the vestiges of the merging process. In fact, these 'satellites' enter, orbit and get progressively stripped of their outer layers while inside the host halo.

It was originally thought that substructures would be quickly erased but the advent of high-resolution $\mathrm{N}$-body simulations revealed that this is not the case (e.g. Moore et al. 1996; Tormen et al. 1998; Klypin et al. 1999). Comparing the computer models with the population of Milky-Way satellites posed several challenges to the $\Lambda$ CDM model (see e.g. Kravtsov 2010; Bullock 2010; Weinberg et al. 2015, for a review). Recent studies have characterized the detailed statistical properties of the surviving satellites (Gao et al. 2011; Ishiyama et al. 2013; Wu et al. 2013) and

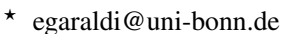

$\dagger$ Member of the International Max Planck Research School (IMPRS) for

Astronomy and Astrophysics at the Universities of Bonn and Cologne shown that they are very sensitive to gas and star-formation physics (e.g. Dolag et al. 2009; Okamoto et al. 2010; Romano-Díaz et al. 2010; Schewtschenko \& Macciò 2011; Di Cintio et al. 2013; Wang et al. 2016; Chua et al. 2016).

This article is the last in a series of three introducing a numerical project named Zooming On a Mob of Galaxies (ZOMG). ZOMG uses a suite of zoom $N$-body plus hydrodynamical simulations to study how the cosmic environment regulates the evolution and properties of galaxy-sized dark-matter haloes as well as of their baryonic content and substructures. It is well known that haloes of the same mass show different clustering properties depending on their formation history, a process usually dubbed 'assembly bias' (Gao et al. 2005; Harker et al. 2006; Zhu et al. 2006; Gao \& White 2007; Dalal et al. 2008). A long-standing challenge in theoretical cosmology has been to understand the origin of this phenomenon, especially for galaxy-sized haloes. Building upon the early work by Hahn et al. (2009), in Borzyszkowski et al. (2017, hereafter paper I), we have shown that haloes stop growing in mass once they are embedded in prominent filaments of the cosmic web that are thicker than the halo diameter. The dark matter (DM) in these haloes preferentially follows tangential orbits due to the gravitational pull of the filament that alters the trajectories of the infalling material before it reaches the halo. Conversely, haloes sitting at the knots of the cosmic web (the regions towards which numerous thin 
filaments converge) grow by accreting material from the surroundings along quasi-radial orbits. The accretion history and the internal dynamics of galaxy-sized haloes are thus intimately related to the halo location within the cosmic web, hence the assembly bias. It is natural to ask whether the fate of the gas component also depend on the halo assembly history and position. In Romano-Díaz et al. (2017, hereafter paper II), we have addressed this question showing that the properties of the central galaxy are largely insensitive to the collapse time of the host, with the exception of the thickness and age of its stellar disc (both increasing for haloes with stalled growth in filaments). Finally, in this work, we investigate the impact of the halo assembly history (and thus of cosmic environment) on the properties of the satellite population.

Several related lines of research have been recently pursued using numerical simulations. Although they have identified the existence of deep interrelationships between halo environment, matter accretion and the final characteristics of the substructures, a clear picture has not yet emerged. The connection between cosmic filaments and the kinematics of substructures has been particularly explored in the literature. Infall along filaments seem to produce groups of subhaloes whose orbital angular momenta align with the halo spin in both possible rotating directions (Lovell et al. 2011). Even the survival lifetime of substructures seem to depend on whether they have been accreted along a filament or not (González $\&$ Padilla 2016). It has been concluded that the ordered accretion of substructures along filaments is the prime reason for the existence of flattened configurations of satellite galaxies which are coherently rotating (Libeskind et al. 2005, 2015; Lovell et al. 2011). Some authors, however, argue that such layouts can only exist when at most two filaments feed the host halo (Ahmed et al. 2017). Others find that thin planes of satellites are only hosted by haloes with very concentrated mass density profiles which form early on when filaments are narrower and accretion is thus more focused (Buck et al. 2015). In general, the spatial distribution of the satellites seem to align (to some extent) with the large-scale distribution of matter surrounding the host halo and even with the halo shape (Shao et al. 2016). In fact, substructures preferentially fall in along the main principal axis of the inertia tensor (Libeskind et al. 2014).

In this paper, we use the high-resolution simulations presented in paper I and paper II to study how the population of satellite galaxies is influenced by the halo assembly history. The article is structured as follows. In Section 2, we describe the main features of the ZOMG simulations and the analysis performed for this work. The detailed time evolution of a few substructures is described in Section 3 while, in Section 4, we study the statistical properties of the satellite populations providing comparisons with previous numerical studies and observations. In Section 5, we tackle the issue of how the process of halo assembly impacts the kinematics and the final spatial configuration of the satellites. A summary of our results and conclusions are presented in Section 6.

\section{NUMERICAL METHODS}

We summarize here the main properties of the ZOMG simulations and discuss the substructure analysis which forms the main focus of the paper. Further details can be found in papers I and II.

The ZOMG project includes a set of high-resolution $N$-body and hydrodynamical simulations that follow structure formation in a model universe with $\Omega_{m}=0.308, \Omega_{\Lambda}=0.692, \Omega_{b}=0.0481$ and $h=0.678$ (Planck Collaboration et al. 2014). Linear density perturbations are characterized by the spectral index $n=0.9608$ and the power-spectrum normalization $\sigma_{8}=0.826$. All simulations cover the same periodic cubic box with a side of $50 \mathrm{~h}^{-1} \mathrm{Mpc}$. Initial conditions are generated at redshift $z=99$ using the MUSIC code (Hahn \& Abel 2011) and employing second-order Lagrangian perturbation theory to shift particles from a uniform Cartesian grid.

To isolate the host haloes of present-day $L_{*}$ galaxies, we make use of a parent $N$-body run containing $512^{3}$ identical particles and select a few objects with masses $M_{\mathrm{h}} \sim$ few $\times 10^{11} h^{-1} \mathrm{M}_{\odot}$ at $z=0$ that we then re-simulate at very high-resolution using the multimass zoom technique. On top of the mass selection, we apply a further criterion based on the assembly history of the haloes that we characterize in terms of the 'collapse time' introduced in Borzyszkowski et al. (2014). In brief, we trace the particles that form a halo at $z=0$ back in time and compute the evolution of their tensor of inertia. Given the inertia ellipsoid, we rigidly rescale it so that it always contains the final mass of the halo and we calculate its volume $V(t)$. This procedure was conceived to follow the evolution of the outermost matter shell forming the halo at $z=0$, the collapse of which ultimately determines the halo assembly time. The function $V(t)$ initially increases due to the Hubble expansion, reaches a maximum (that can be used to define the epoch of turnaround) and generally decreases afterwards until it starts oscillating around a constant value indicating virialization (actual examples are shown in Fig. 1 of paper I). The collapse time of a halo, $t_{\mathrm{c}}$ (or the corresponding redshift $z_{\mathrm{c}}$ and expansion factor $a_{\mathrm{c}}$ ), is defined as the moment at which the volume stabilizes (see paper I for details regarding the practical implementation of this calculation). Haloes with fixed mass in the parent run show a broad range of collapse times. To maximize the differences among the re-simulated haloes and better recognize the effects of their assembly histories, we only pick objects in the tails of the distribution. For the $N$-body (DMonly) runs (see paper I), we thus randomly pick 5 'stalled' $\left(z_{\mathrm{c}}>1\right)$ and 2 'accreting' $\left(z_{\mathrm{c}} \lesssim 0\right)$ objects among the haloes of the selected mass identified in the parent simulation at $z=0$. For the much more time consuming hydrodynamical simulations (see paper II), instead, we only consider 2 haloes for each class. We adopt the nomenclature of papers I and II where each re-simulated halo is named after an ancient god, sharing the initial letter of its name with the class it belongs to (i.e. ' $\mathrm{S}$ ' for stalled and ' $\mathrm{A}$ ' for accreting haloes).

The re-simulations are carried out using a modified version of the tree-particle-mesh smoothed particle hydrodynamics (SPH) code PGADGET-3 (Springel 2005). Our hydrodynamic runs include radiative cooling, star formation and stellar feedback, galactic winds, a multi-phase interstellar medium (Springel \& Hernquist 2003) and an ultraviolet background active from $z \sim 11$ that reionizes hydrogen in the intergalactic medium by $z \sim 6$ (Haardt \& Madau 2001). In all cases, we achieve an effective resolution of $4096^{3}$ computational elements in the region of interest (roughly extending up to three times the halo radius), corresponding to particle masses of $m_{\mathrm{DM}}=1.31 \times 10^{5} h^{-1} \mathrm{M}_{\odot}$ and $m_{\text {gas }}=2.43 \times 10^{4} h^{-1} \mathrm{M}_{\odot}$ for DM and gas, respectively. Each gas particle can experience up to two episodes of star formation, in each of which a mass $m_{\text {gas }} / 2$ is converted into a stellar particle. Our runs extend to $z=0$. We save a few snapshots at early times plus one every $20 \mathrm{Myr}$ after redshift $z=9$, for a total of 682 output files.

We identify gravitationally bound objects and their substructures using the Amiga HaLo Finder (AHF, Gill et al. 2004; Knollmann \& Knebe 2009). This software initially defines haloes as spherical regions with a mean matter density of 200 times the critical density of the universe, $\rho_{\mathrm{c}}(z)$, and then iteratively purges them of the (unbound) particles that move faster than 1.5 times the escape 


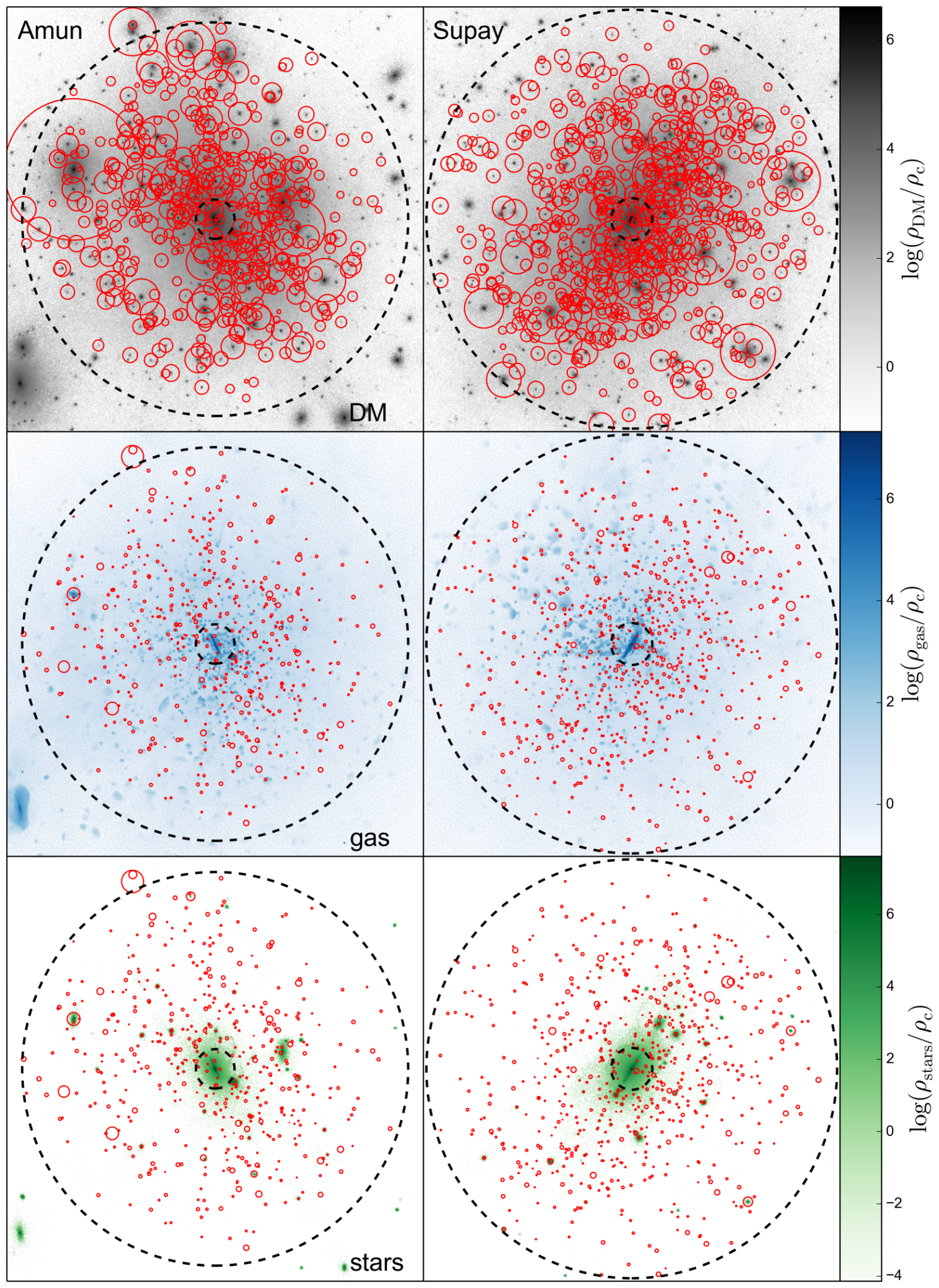

Figure 1. Dark-matter, gas and stellar distributions (from top to bottom) for two of our re-simulated haloes at $z=0$. Each panel shows the projection along one axis of the simulation particles contained within a cube of side $310 \mathrm{~h}^{-1} \mathrm{kpc}$ centred on the corresponding halo. Particles are colour-coded according to the local density computed using a standard SPH-like kernel with 64 neighbours (of the same species), normalized by the critical density of the universe $\rho_{\mathrm{c}}$. The outer and inner dashed circles indicate the halo radius $R_{\mathrm{h}}$ and the radius of the central galaxy $R_{\mathrm{g}}$, respectively. Substructures are highlighted with solid red circles whose radius reflects the subhalo radius found by AHF (top panels) or the location of the maximum circular velocity (middle and bottom panels). Note that dense DM clumps that are not surrounded by a red circle are located outside the halo radius although they might give the impression to be within $R_{\mathrm{h}}$ due to projection effects. 
Table 1. Properties of the re-simulated haloes at $z=0$. From left to right, the columns give: the name, the mass $\left(M_{\mathrm{h}}\right)$, the radius $\left(R_{\mathrm{h}}\right)$, the scale radius $\left(r_{\mathrm{s}}\right)$ obtained fitting the radial mass-density profile with the Navarro-Frenk-White (NFW) formula (Navarro et al. 1997), the expansion factor at collapse time $\left(a_{\mathrm{c}}\right)$, the total number of substructures $\left(N_{\mathrm{sh}}\right)$, the number of substructures with gas $\left(N_{\mathrm{gas}}\right)$, the number of substructures with stars $\left(N_{*}\right)$, the fraction of $M_{\mathrm{h}}$ in substructures $\left(f_{\mathrm{sh}}\right)$, the mean fraction of the baryonic content of substructures identified at $z=2$ that migrates to the central galaxy by $z=0\left(\left\langle f_{\text {disc }}\right\rangle\right)$ and the colour associated to the halo throughout the paper.

\begin{tabular}{|c|c|c|c|c|c|c|c|c|c|c|}
\hline $\begin{array}{l}\text { Halo } \\
\text { name }\end{array}$ & $\begin{array}{c}M_{\mathrm{h}} \\
{\left[10^{11} h^{-1}\right.}\end{array}$ & $\begin{array}{c}R_{\mathrm{h}} \\
\left.\mathrm{M}_{\odot}\right]\left[h^{-1} \mathrm{kpc}\right]\end{array}$ & $\begin{array}{c}r_{\mathrm{s}} \\
{\left[h^{-1} \mathrm{kpc}\right]}\end{array}$ & $a_{\mathrm{c}}$ & $N_{\mathrm{sh}}$ & $N_{\text {gas }}$ & $N_{*}$ & $f_{\mathrm{sh}}$ & $\left\langle f_{\text {disc }}\right\rangle$ & colour \\
\hline Abu & 4.1 & 151 & 3.13 & 0.968 & 382 & 4 & 52 & 0.109 & 0.03 & \\
\hline Amun & 3.5 & 144 & 7.41 & $>1.00$ & 477 & 4 & 73 & 0.100 & 0.05 & \\
\hline Supay & 4.3 & 153 & 5.05 & 0.404 & 647 & 3 & 97 & 0.067 & 0.09 & \\
\hline Siris & 2.3 & 124 & 3.02 & 0.333 & 228 & 1 & 32 & 0.067 & 0.03 & \\
\hline
\end{tabular}

velocity. The halo radius, $R_{\mathrm{h}}$, and mass, $M_{\mathrm{h}}$, are defined using the smallest sphere enclosing all the bound particles. We conventionally identify the 'central galaxy' of each halo with the innermost region of radius $R_{\mathrm{g}}=0.1 R_{\mathrm{h}}$ (e.g. Scannapieco et al. 2012). Following Diemer \& Kravtsov (2014), we also consider a second definition for the halo boundary by locating a sharp steepening of the radial mass-density profile. Adhikari et al. (2014) and More et al. (2015) argued that such radius correspond to the first apocentre of recently accreted matter and therefore dubbed it as the 'splashback radius', $R_{\text {spl }}$. Contrary to the halo radius, $R_{\text {spl }}$ is parameter free and does not suffer from pseudo evolution due to change of $\rho_{\mathrm{c}}$ with time.

We use the built-in function of AHF to construct halo merger trees. The main progenitor of a halo is selected maximizing the merit function $N_{i \cap j}^{2} / N_{i} N_{j}$, where $N_{i}$ and $N_{j}$ denote the number of particles in the progenitor and the descendant in two consecutive snapshots and $N_{i \cap j}$ is the number of particles they share.

AHF automatically detects substructures as density peaks within a main halo. Their edge is initially determined as the minimum of the radial density profile and then adjusted to the radius of the smallest sphere enclosing all bound particles. Note that we do not consider higher levels of (nested) substructures (i.e. subsubhaloes are not distinguished from their host subhaloes). Substructures are dynamic entities that continuously accrete and loose material. When studying their evolution, we follow all the particles they are made of at the time they reach their maximum mass (typically, right before entering the host).

Fig. 1 gives a visual impression of two of our re-simulated haloes and of their substructures. The main properties of the four haloes analyzed in this paper are listed in Table 1. These data are extracted from the hydrodynamic simulations, corresponding results for the $N$-body runs can be found in Table 1 of paper I.

\section{SATELLITE EVOLUTION}

In this section, we exploit the excellent time- and mass-resolution of the ZOMG simulations to describe the evolutionary path of a few typical substructures. This study helps the reader recognize the complex phenomenology of satellite-host interactions and us introduce several key concepts that will be used in the remainder of the paper. Ultimately, we determine the amount of material that satellites shed to their host halo and its central galaxy.

\subsection{Trajectories and dynamics}

We conventionally define the accretion redshift of a satellites, $z_{\mathrm{ac}}$, by identifying the instant when, for the first time, AHF associates it with a host halo. The subsequent fate of the satellite is regulated by the interplay between several physical mechanisms (e.g. Binney \& Tremaine 2008; Mo et al. 2010; Mayer 2010, and references therein). Gravitational interactions between the satellite and the diffuse material that makes up the host produce a net drag known as dynamical friction. As a result, the satellite looses energy and angular momentum and its orbit decays towards the central region of the host where the gravitational potential reaches its minimum value. Along the way, the satellite constantly looses matter from its outer parts due to the action of tidal forces (tidal stripping). The ejected material initially forms leading and trailing streams stretching for large distances compared with the core of the satellite. Later on, these tidal tails evolve into approximately spherical shells and eventually phase-mix with the diffuse component of the host halo. In the CDM scenario, most satellites accrete on to their hosts following highly eccentric orbits and thus experience time-varying tidal forces. At each pericentric passage, when tides become particularly strong, the satellite expands, gains kinetic energy and rearranges its internal structure (tidal heating). Its lower binding energy makes it prone to further mass loss via tidal stripping (and ram pressure stripping for the gas component). Since the orbital decay rate depends on the satellite mass, all these effects are highly interconnected. The detailed evolution thus depends on the inital mass and concentration as well as the orbital parameters of the satellite.

In order to provide a few representative examples, in Fig. 2 we consider two rather massive substructures extracted from our simulations. Although they both have $z_{\mathrm{ac}} \approx 2$, one of them survives till the present time while the other is completely disrupted (meaning that AHF cannot identify it any longer at late times against the background of the host halo). For this reason, we dub them S-Sat and D-Sat, respectively. S-Sat (shown on the left-hand side) is part of Supay while D-Sat (displayed on the right-hand side) is hosted by Amun, although this is not important as similar examples are present in every re-simulated halo. In the top panels, we show the distance of the satellites from the centres of the corresponding hosts as a function of time. The curves start well before the satellites first 'enter' their host and follow their radial trajectories until the present time. S-Sat follows a very eccentric orbit and only experiences two pericentric passages while D-Sat describes a series of fast-decaying orbits and completes many pericentric passages. At accretion, SSat and D-sat are characterized by similar relative velocities with respect to the host although the impact parameter of D-Sat is three times larger than for S-Sat.

In the central panels, we show the time evolution of the satellite mass in DM (green), gas (blue) and stars (red). At accretion, SSat has a (total) mass of $M_{\mathrm{ac}}=2.9 \times 10^{8} h^{-1} \mathrm{M}_{\odot}$ which progressively reduces to $M_{\mathrm{sh}}(z=0)=8.7 \times 10^{7} h^{-1} \mathrm{M}_{\odot}$. D-Sat is initially much more massive, with $M_{\mathrm{ac}}=1.3 \times 10^{9} h^{-1} \mathrm{M}_{\odot}$. The stellar and DM masses remain fairly constant until late times when the satellites reach the densest regions of the host haloes and are tidally stripped. 

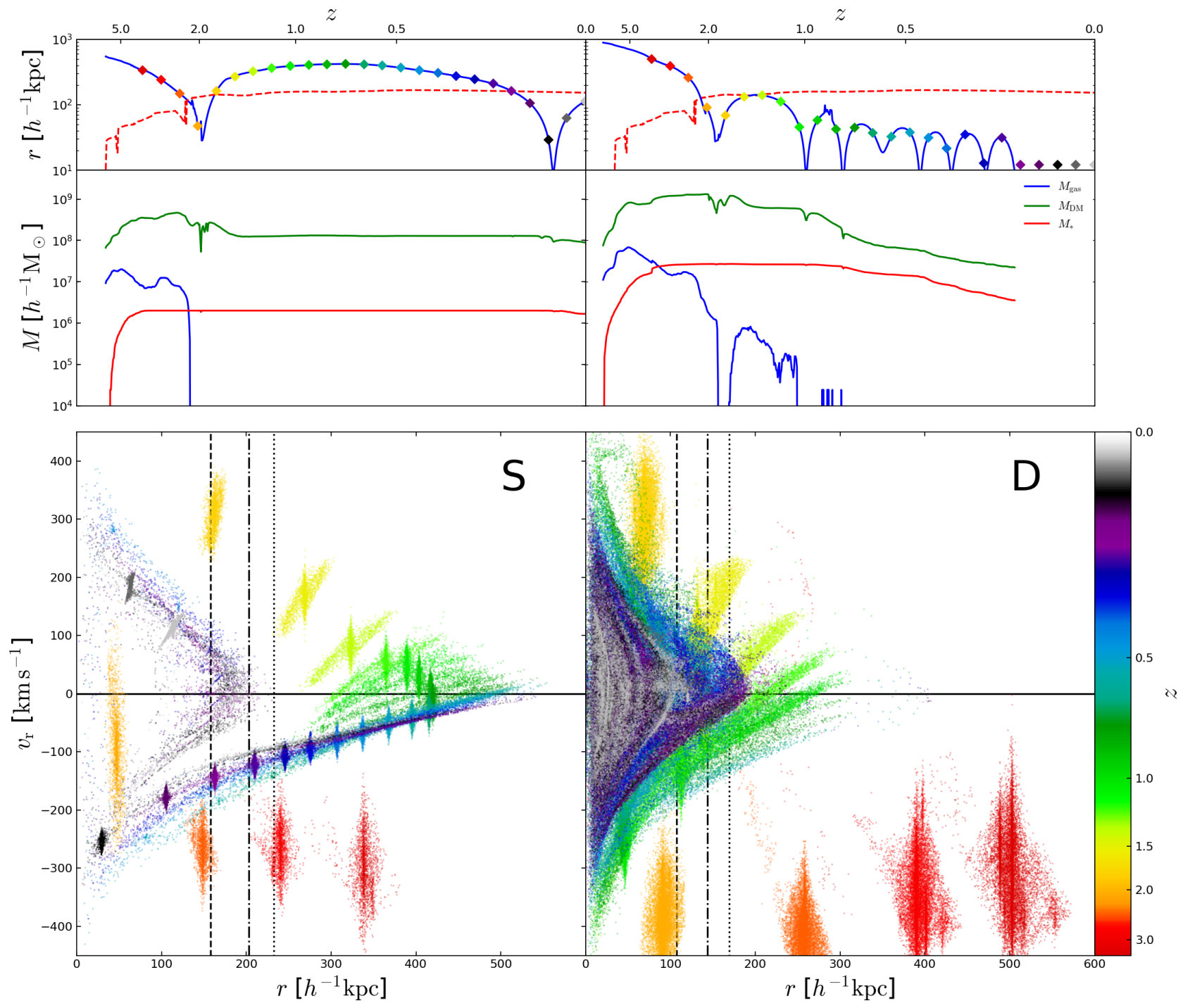

Figure 2. Evolution of different properties in two of the most massive subhaloes in our simulation suite (the left one belongs to Supay while the right one is part of Amun). Top: evolution of the distance $r$ from the host. The dashed red line denotes the host radius. The diamond symbols indicate the redshift of the steps plotted in the bottom panels. Middle: evolution of the DM, gas and stellar mass of the substructure (green, blue and red line, respectively). Bottom: radial phase space distribution of all particles. The colour encodes the redshift, in steps of $500 \mathrm{Myr}$. The substructure in the left panel has survived until $z=0$ while the one in the right panel has been totally disrupted. The halo radius, the splashback radius and its predicted (median) value for haloes with the same mass and accretion rate (following More et al. 2015) are shown at the redshift of the first apocentre using dashed, dot-dashed and dotted lines respectively.

On the other hand, the gas component follows a very different evolutionary path. Even before $z_{\mathrm{ac}}$, gas is stripped off the (yet to be) satellites. This is due to the combined action of two mechanisms: heating caused by the ultraviolet background radiation and interactions with an increasing environment density. After the reionization of the intergalactic medium is completed at $z \sim 6$, photo-heating affects low-density gas which is not self-shielded. As a consequence, the gas reservoir of haloes that are below the atomic cooling mass limit (Doroshkevich et al. 1967; Rees 1986) can be completely depleted (Gnedin 2000; Okamoto et al. 2008). Simultaneously, as the low-mass haloes approach their final more-massive host, they find themselves in denser and denser environments of the cosmic web and can loose their gas via ram pressure stripping Benítez-Llambay et al. (2016). Both S-Sat and D-Sat are massive enough to retain a substantial amount of gas at $z_{\mathrm{ac}}$. However, after they accreted on to their hosts, they are completely deprived of gas within the first few pericentric passages (see also Helmi \& White 1999).

A more detailed view of the fate of a substructure is provided by the radial phase space plots in the bottom panels of Fig. 2. Here we show the location of the simulation particles (DM, gas and stars) that form S-Sat and D-Sat from $z=3.5$ to $z=0$ with time steps of $500 \mathrm{Myr}$. The particles are colour-coded based on redshift and, to facilitate understanding, symbols of the corresponding colours are also shown in the top panel. The effect of tidal interactions is clearly noticeable in the bottom-left panel. Particles start being stripped off $\mathrm{S}$-Sat during its first pericentric passage. The debris form tidal tails that eventually fall on to the host along a very extended stream. At $z=0$, the original subhalo has been split in three different components: the material stripped during the first orbit lying inside the halo; the recently-disrupted material, as distant as $400 h^{-1} \mathrm{kpc}$, being slowly accreted by the main halo, and the surviving subhalo 

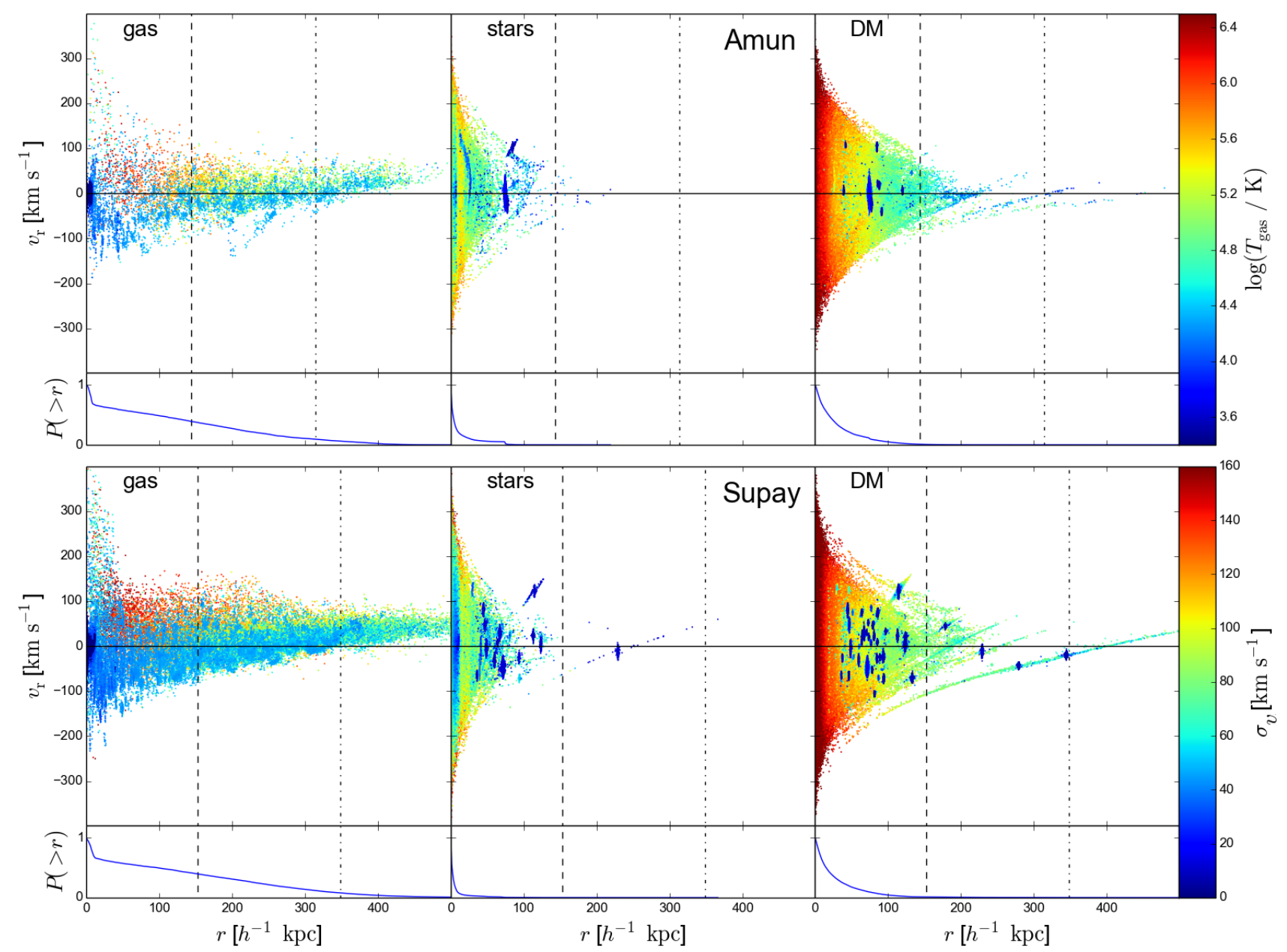

Figure 3. Radial phase space distribution at $z=0$ of particles of different species belonging to the 100 most massive subhaloes identified at $z=2$ in Amun (top) and Supay (bottom). The particles are colour-coded according to the temperature of the gas (left) and the velocity dispersion of stars (middle) and DM (right). The inset on the bottom of each panel shows the cumulative probability distributions of finding a particle at a distance larger than a given radius. Note that the cold gas with high (positive) radial velocities at very small radii is due to the numerical treatment of supernova feedback in the central galaxy.

orbiting around the host. Each pericentric passage ends up generating a dynamically coherent structure in which positions and velocities are strongly correlated. One of these 'tidal caustics' (e.g. Mohayaee \& Shandarin 2006) is clearly noticeable in the radial phase-space diagram for S-Sat as a symmetric distribution of loose particles with a uniform colour and a characteristic bell shape. Note that tidal caustics have a finite width and density (contrary to genuine caustics that are generated by a perfectly cold distribution of particles with the same energy). The build up of tidal streams and caustics is particularly evident in the bottom-right panel. Due to its larger mass, D-Sat experiences stronger dynamical friction than SSat. Consequently, it orbits much closer to the centre of the host and with a shorter period, resulting in a rapid disruption. A series of tidal caustics corresponding to the multiple orbits of the satellite are in fact noticeable in the phase-space diagram of the loose material at $z=0$.

Fig. 2 shows that, after its first pericentric passage, S-Sat reaches a distance of $\sim 415 h^{-1} \mathrm{kpc}$ from its host halo while some of the stripped debris stretch out to more than $500 \mathrm{~h}^{-1} \mathrm{kpc}$ of separation. Note that $\mathrm{S}$-Sat travels across its first apocentre at $z_{\mathrm{ap}}=0.8$ when the radius of Supay is $R_{\mathrm{h}}=160 h^{-1} \mathrm{kpc}$ (dashed line in the bottom-left panel). This phenomenon is quite common (see also Ludlow et al. 2009, and Fig. 8 in Paper I) and makes it interesting to compare the apocentric distance of the satellite with the splashback radius of the host halo which is generally assumed to mark its physical outer boundary. We first estimate $R_{\text {spl }}$ by locating the minimum of the logarithmic derivative of the spherically averaged mass density profile (More et al. 2015). Our result at $z_{\text {ap }}$ is shown with a dot-dashed line. Since numerical derivatives are noisy, we also make use of the SHELLFISH code (Mansfield et al. 2016) to reconstruct the full three-dimensional shape of the splashback surface and derive $R_{\mathrm{spl}}$ as the radius of the sphere with the same enclosed volume. In all cases, this estimate differs by less than 4 per cent from the previous one. Finally, we obtain a third value for $R_{\text {spl }}$ by using a fit to the median splashback radius of haloes with a given mass and accretion rate (averaged over time scales comparable with the halo crossing time, see equation (5) in More et al. 2015) and providing the appropriate input for S-Sat. The resulting $R_{\text {spl }}$ (dotted line) is always larger than our previous estimates but still substantially lower than the apocentric distance of S-Sat. Although we have presented in detail only one specific example, the same conclusions can be reached after studying the evolution of a very large number 
of satellites. We find that the first apocentric distance lies beyond the splashback radius for nearly 40 per cent of the satellites, regardless of the host halo. It is worth stressing that two distinct concepts have been mixed up under the name of splashback radius (Adhikari et al. 2014; More et al. 2015). In practical calculations, $R_{\mathrm{spl}}$ is defined as the location at which the halo mass-density profile presents a sudden steepening of its slope. On the other hand, based on spherical models of collisionless secondary infall, $R_{\mathrm{spl}}$ is interpreted as the position of the outermost density caustic and, by extension, as the radius at which newly accreted matter reaches its first apocentre. Our results indicate that, in realistic cases, these two concepts do not perfectly match and the practical definition of $R_{\text {spl }}$ needs to be further refined in order to make them compatible. A step forward in this direction has been made by Diemer (2017) who used $\mathrm{N}$-body simulations to investigate the relation between $R_{\text {spl }}$ and the first apocentric distances of DM particles that are not part of substructures. Our complementary study, instead, follows the orbits of satellite galaxies in haloes of lower mass. Further understanding is also required to optimise the strategy for the observational detection of the splashback radius in galaxy clusters using their member galaxies (More et al. 2016; Baxter et al. 2017).

\subsection{Shed material}

We now focus on the material that was originally locked in substructures and later became part of the host halo and its central galaxy (this study completes the analysis presented in paper II). For example, in Fig. 3, we consider the 100 most massive substructures identified at $z=2$ in Amun (top) and Supay (bottom) and plot the radial phase space distribution of their original constituents (gas, stars and DM) at $z=0$ (similar conclusions can be drawn selecting Abu and Siris, as well as halving or doubling the sample of substructures). Particles are colour-coded according to their temperature (for the gas) or velocity dispersion (for stars and DM) computed using the 64 nearest neighbours of the same species. The inset on the bottom of each panel shows the cumulative probability distribution $P(>r)$ of finding a particle of the given species at distances larger than $r$. The phase-space distribution of the gas extends to larger radii with respect to the bulk of the collisionless components. While stars and DM show clumps associated with surviving satellites, virtually all the gas has been stripped off of the substructures. Shock-heating and feedback mechanisms have ejected a good fraction of this gas outside the main halo from where it can rain back in once it has cooled down. However, the cooling time can be quite long depending on the local metallicity and density. It is worth stressing that only half of the gas that was part of substructures at $z=2$ is still found within $R_{\mathrm{h}}$ at $z=0$ (see also the discussion about recycled material in paper II). This effect might not be accurately captured by certain 'semi-analytic' models of galaxy formation that make too simplistic assumptions concerning gas stripping from satellites and stellar feedback (see also Hirschmann et al. 2012).

Baryonic material that was at first part of substructures also migrates to the central galaxy and its disc. By approximating the latter as a cylinder with height and radius corresponding to three times the scale values derived in paper II, we determine the fraction $f_{\text {disc }}$ of the original baryonic mass of a $z=2$ substructure that is found in the galaxy at $z=0$. This quantity increases with $M_{\mathrm{sh}}$ since massive subhaloes are more affected by dynamical friction and retain a larger gas reservoir until disruption. Although $f_{\text {disc }}$ shows a lot of scatter, its mean value for each of the ZOMG haloes is rather small, ranging between 3 and 9 per cent (see Table 1). The halo

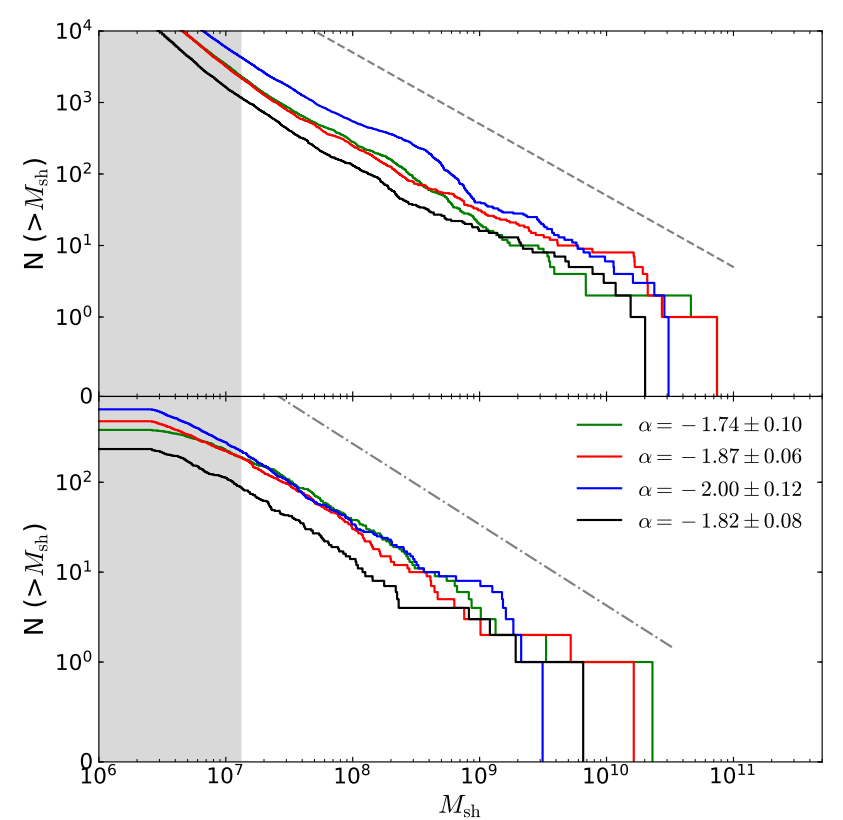

Figure 4. Unvolved (top) and evolved (bottom) cumulative subhalo mass functions. The gray vertical shaded region corresponds to subhalo masses smaller than $100 \mathrm{DM}$ particles. The dashed and dot-dashed lines are used as a reference and correspond to a power law with slope $\beta=-1$ and $\alpha+1=$ -0.9 , respectively. In the bottom panel the slope of the best-fitting power law for each host are reported.

assembly time does not seem to have an influence on $\left\langle f_{\text {disc }}\right\rangle$. However, this quantity is substantially larger for Amun and Supay that host central galaxies with a prominent disc component (see paper II). This is not only reflecting the larger disc size but is also a consequence of the deeper potential well that alters the orbits of the satellites (Garrison-Kimmel et al. 2017). Consistently, the (baryonic) mass fraction of the present-day disc that was inside substructures at $z=2$ amounts to 32 and 8 per cent for Supay and Amun, respectively, while is smaller for Siris ( 3 per cent) and in particular for Abu ( 0.5 per cent). Note that only a few massive substructures with large $f_{\text {disc }}$ determine the value for Supay.

\section{SUBHALO STATISTICS}

After having described the phenomenology and the main physical mechanisms of satellite evolution, we investigate the statistical properties of the surviving substructures. The goal of this section is twofold. First, we show that our simulations are in agreement with many observations and previous numerical studies. At the same time, however, we try to isolate possible distinctive features characterizing stalled and accreting haloes. Since observation of satellites are only possible in the local universe, we exclusively present results at $z=0$ (unless explicitly stated otherwise).

\subsection{Mass and radial distribution}

In the bottom panel of Fig. 4 we show the distribution by mass of satellites in each of the ZOMG haloes (within $R_{\mathrm{h}}$ and at $z=0$, commonly dubbed subhalo mass function, or sHMF) together with 


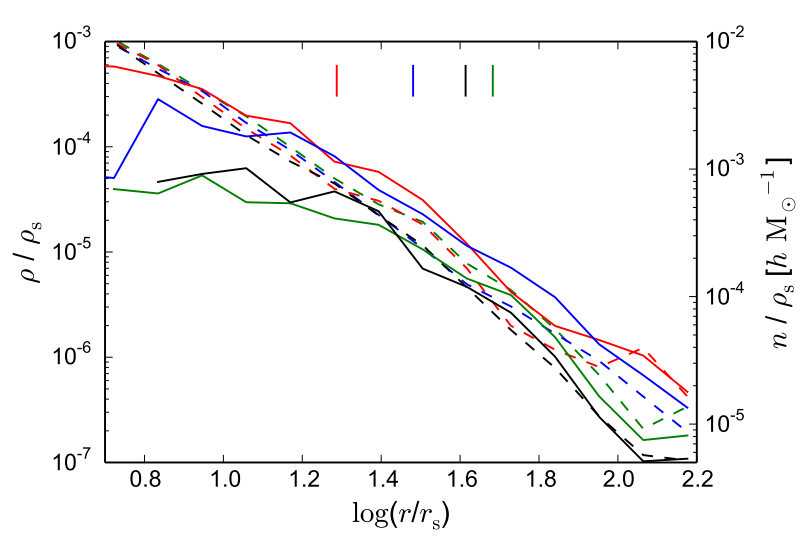

Figure 5. Radial distributions of substructures (solid) and matter (dashed) within and around the ZOMG haloes at $z=0$. Note the different vertical scale for the two profiles, which are rescaled by the best-fitting value of $\rho_{\mathrm{s}}$. Similarly, $r$, the distance from the host centre, is normalized by the scale radius $r_{\mathrm{s}}$. Vertical segments indicates the radius of each halo.

the slope of the corresponding best-fitting power law of the form

$$
\frac{\mathrm{d} N\left(M_{\mathrm{sh}}\right)}{\mathrm{d} M_{\mathrm{sh}}} \propto M_{\mathrm{sh}}^{\alpha}
$$

(note that, while we show the cumulative sHMF, we perform the fit using the differential distribution of $M_{\mathrm{sh}}$ in order to avoid highly correlated errors). In the fit, we weigh the binned counts according to their Poisson errors and, to limit incompleteness due to resolution effects, we only consider satellites containing at least 100 DM particles (this mass limit is indicated in Fig. 4 with a vertical shaded region). The best-fitting slopes for the different ZOMG haloes are compatible within the errorbars (the actual values are reported in Fig. 4). Overall, they are in good agreement with the value of $\alpha \simeq-1.9$ generally found in high-resolution $N$-body (Klypin et al. 1999; Moore et al. 1999; Ghigna et al. 2000; Springel et al. 2008; Gao et al. 2012; Cautun et al. 2014; Hellwing et al. 2016) and hydrodynamical (Romano-Díaz et al. 2010; Sawala et al. 2013, 2017) simulations (dot-dashed line). This is also consistent with observational estimates based on strong (Vegetti et al. 2014, for the substructure in galaxy-sized haloes) and weak (Okabe et al. 2014, for the Coma cluster) gravitational lensing.

In the top panel of Fig. 4, we show the probability distribution of the satellite masses at accretion for each ZOMG halo. This unevolved sHMF is usually averaged over all main haloes present at a given time and includes the satellites accreted at any previous time. Numerical studies found it can be well approximated by a power-law

$$
\frac{\mathrm{d} N\left(>M_{\mathrm{ac}}\right)}{\mathrm{d} M_{\mathrm{sh}}} \propto M_{\mathrm{sh}}^{\beta}
$$

with slope $\beta=-1$ (Giocoli et al. 2008; Springel et al. 2008; Han et al. 2016). Such relation (dashed line) is in good agreement with our results for single haloes.

Within $R_{\mathrm{h}}$, the spherically averaged mass density profiles of the ZOMG haloes are well described by the NFW function

$$
\rho(r)=\frac{4 \rho_{\mathrm{s}}}{\left(r / r_{\mathrm{s}}\right)\left[1+\left(r / r_{\mathrm{s}}\right)^{2}\right]}
$$

where $r_{\mathrm{s}}$ and $\rho_{\mathrm{s}}=\rho\left(r_{\mathrm{s}}\right)$ denote a characteristic radius and density. This is shown by the dashed lines in Fig. 5 where we have appropriately rescaled the horizontal and vertical axes so that the different

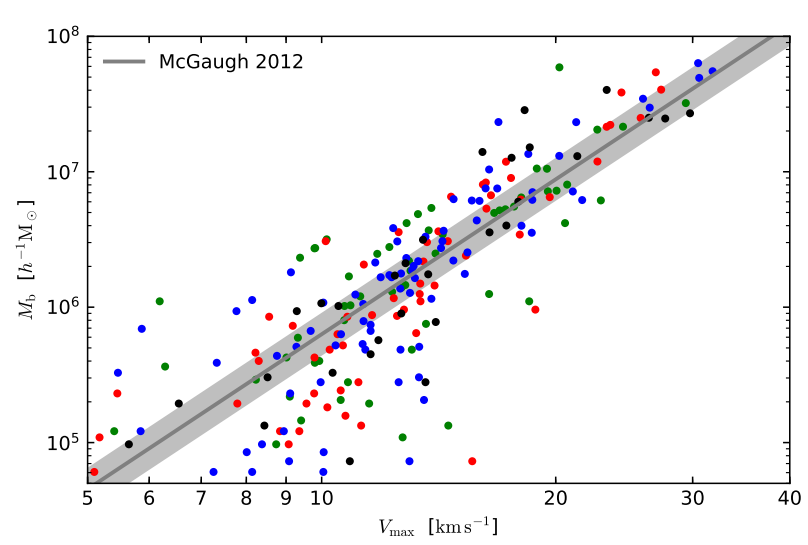

Figure 6. Baryonic Tully-Fisher relation for the ZOMG subhalo populations. The solid line and the shaded region denote the best-fitting power law and the maximum estimated scatter for the observational data, respectively (McGaugh 2012).

curves should coincide if the mass profiles exactly follow equation (3). The corresponding values of $R_{\mathrm{h}}$ are indicated by vertical segments (see also Table 1). We now compare the radial distribution of the satellites in accreting and stalled haloes at $z=0$ (solid lines in Fig. 5). All the hosts show the same pattern: the satellite distribution traces the matter profile for $r \gtrsim 0.5 R_{\mathrm{h}}$ but flattens out at smaller radii where substructures are more easily disrupted. Similar findings have been originally reported for cluster-sized haloes (Nagai \& Kravtsov 2005; Gao et al. 2012) and, later, on galaxy scales based on dark-matter-only (e.g. Han et al. 2016) and hydrodynamical simulations (e.g. Libeskind et al. 2007; Schewtschenko \& Macciò 2011; Zhu et al. 2016).

\subsection{Gas and stellar content}

We now delve into the analysis of the stellar and gas content of substructures. In Fig. 6, we plot the maximum circular velocity, $V_{\max }$, of a subhalo against its total baryonic mass, $M_{\mathrm{b}}$. Observationally, these quantities are tightly correlated and the corresponding powerlaw relation is known as the baryonic Tully-Fisher (BTF) relation (Tully \& Fisher 1977; Walker 1999; McGaugh et al. 2000). Since McGaugh (2012) showed that central and satellite galaxies follow consistent relations, we compare our data to their fit for both populations (solid gray line). The simulated substructures are in good agreement with the observed mean relation and no difference is noticeable between the different ZOMG haloes. It is worth noticing that the simulations overpredict the scatter in the BTF relation, especially at low $V_{\max }$. This is a long-known issue with the standard scenario of galaxy formation (e.g. Dutton 2012 and Lelli et al. 2016, but see Sales et al. 2017 and Sorce \& Guo 2016).

In Fig. 7 we show scatterplots of the 1D stellar velocity dispersion (top) and the stellar mass fraction (bottom) against the total mass of the substructures are shown. The four populations are well mixed and no segregation with $z_{\mathrm{c}}$ is found. In the common mass range, they match data from Milky Way (MW) dwarf spheroidal satellites (Wolf et al. 2010; Parry et al. 2012, black triangles).

Only a minority of the substructures have a stellar counterpart (see e.g. the eighth column of Table 1 and Fig. 1) which typically formed between redshift 5 and 6 . Most satellites, in fact, are below the atomic cooling mass limit and are completely sterilized during the epoch of reionization. Nevertheless, a significant number 


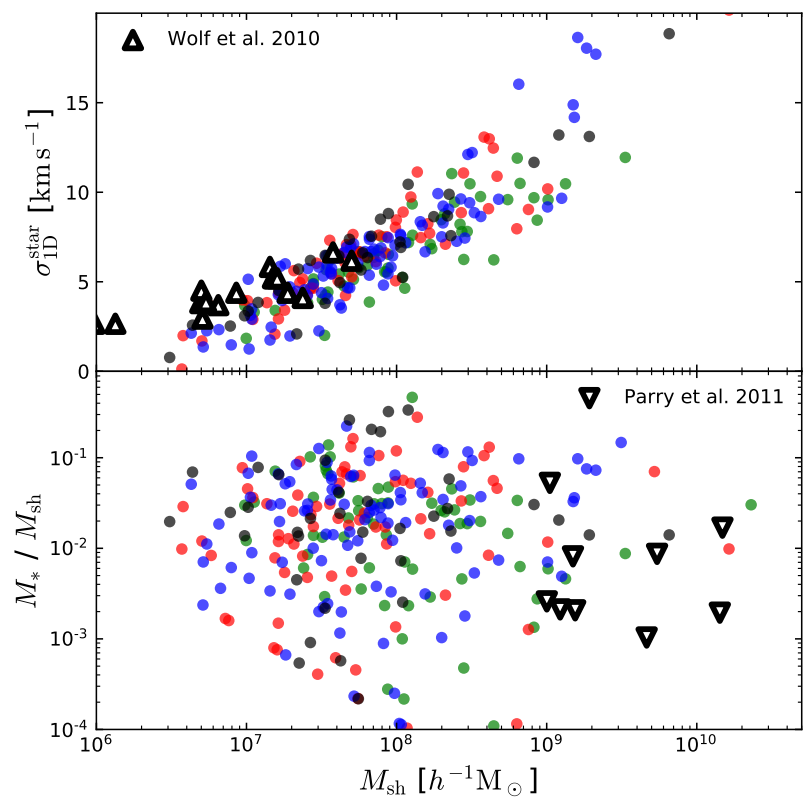

Figure 7. Top: $1 \mathrm{D}$ velocity dispersion for stellar particles $\left(\sigma_{1 \mathrm{D}}^{\text {star }}\right.$, computed assuming isotropy) as a function of subhalo mass. The triangles represent data from Wolf et al. (2010) for Milky Way dwarf spheroidal satellites. Bottom: fraction of mass in stars as a function $M_{\mathrm{sh}}$. Black triangles show data from Milky Way satellites (Parry et al. 2012).

of massive and concentrated subhaloes is able to retain some gas that, sooner or later, contributes to the gas reservoir of the host halo (see Fig. 3) and, possibly, of its central galaxy (paper II). Notably, a relevant portion of the satellites containing some gas at $z=0$ have not just accreted on to their hosts (in some cases $z_{\mathrm{ac}} \sim 1$ ). Overall, the fraction of substructures with gas in the ZOMG project is consistent with previous hydrodynamical simulations of clustersized haloes (e.g. Tormen et al. 2004; Dolag et al. 2009). Comparing with observations, we note that the gas content measured in Milky Way satellites ${ }^{1}$ (Grcevich \& Putman 2009; McConnachie 2012; Spekkens et al. 2014; Westmeier et al. 2015) is well below our mass resolution and thus compatible with our gas-naked substructures.

Finally, we assess the presence of star-forming satellites at $z=0$, defined as substructures containing at least two stellar particles formed in the last $200 \mathrm{Myr}$. Interestingly, they are found only in accreting haloes (Amun contains two of them and Abu one). In particular, they are the most massive subhaloes at $z=0$ $\left(M_{\mathrm{sh}} \gtrsim 5 \times 10^{9} h^{-1} \mathrm{M}_{\odot}\right)$ although their stellar mass is relatively low $\left(M_{*, \mathrm{sh}} \approx 10^{6} h^{-1} \mathrm{M}_{\odot}\right)$. They have already experienced one or two pericentric passages and their star-formation rates (SFR) lie in the range $4 \times 10^{-3}<\mathrm{SFR} \lesssim 1.1 \times 10^{-2} \mathrm{M}_{\odot} \mathrm{yr}^{-1}$. Interestingly, a satellite with similar characteristics (in terms of mass and number of pericentric passages) is hosted by Supay and did not experience any star formation in the last $200 \mathrm{Myr}$. At late times, accreting haloes increase their mass at a faster rate than stalled haloes (paper I). Therefore, they are more likely to host substructures that are

\footnotetext{
1 An obvious exception is provided by the Magellanic Clouds. However, since similar configurations are rare in the $\Lambda \mathrm{CDM}$ cosmology (e.g. Liu et al. 2011), it is not surprising that we do not find any in our relatively small simulation suite.
}

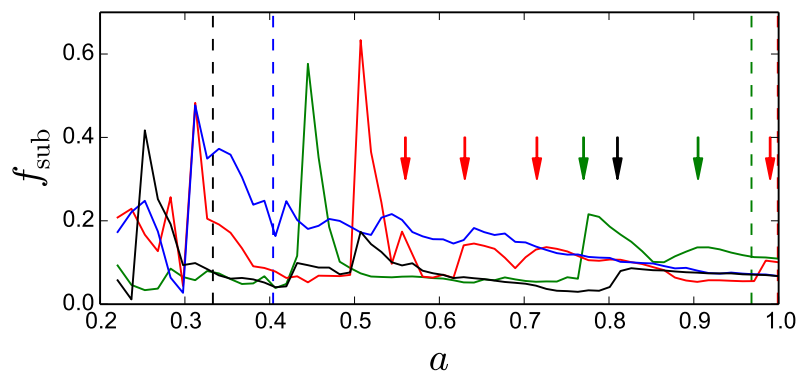

Figure 8. Fraction of the host mass contained in resolved substructures $\left(f_{\mathrm{sh}}\right)$ as a function of expansion factor $a$ for the ZOMG haloes. Dotted lines denotes the $a_{\mathrm{c}}$ of the host. Arrows highlight when the sharp increase in $f_{\mathrm{sh}}$ can be attributed to a massive merger (shown only for $a>0.5$ ).

massive enough to sustain star formation up to $z=0$. We provide further evidence supporting this conjecture in section 4.3.

\subsubsection{Galactic conformity}

The term 'galactic conformity' denotes the tendency of neighbouring galaxies to exhibit similar colours and SF properties. Originally, conformity was detected between galaxies in a single DM halo (Weinmann et al. 2006) although the signal might extend well beyond the virial radius of the host (Kauffmann et al. 2013). In particular, it has been shown that passive (star-forming) central galaxies tend to be surrounded by passive (star-forming) satellites. This trend becomes more and more prominent with decreasing mass of the host halo (e.g. Weinmann et al. 2006; Knobel et al. 2015; Paranjape et al. 2015). In order to extend the theoretical predictions to lower halo masses, we check if the specific SFR (sSFR, i.e. the SFR per unit stellar mass) of our satellite galaxies reflects the value found for the primary galaxy in each ZOMG halo. We follow the strategy of Weinmann et al. (2006) and classify our galaxies as 'quenched' or 'star forming' using a threshold value for the sSFR of $10^{-10} \mathrm{yr}^{-1}$. According to this criterion, all the central and satellite galaxies in our simulations are labelled as quenched at $z=0$, although three of the primaries lie on the star-forming main sequence (see Fig. 17 in paper II). For the satellites, the classification is independent of the stellar masss, the time interval used to evaluate the SFR and the distance from the central galaxy. In fact, the bulk of the satellites do not experience any SF in the last few Gyr. We conclude that the small ZOMG sample shows perfect conformity.

\subsection{Satellite mass fraction}

We compute the fraction of $M_{\mathrm{h}}$ contributed by (resolved) subhaloes, i.e. $f_{\mathrm{sh}} \equiv \sum_{i} M_{\mathrm{sh}, i} / M_{\mathrm{h}}$. At $z=0$, in accreting haloes this quantity is 1.6 times larger than in their stalled counterparts (see Table 1) and its evolution is shown in Fig. 8 as a function of the expansion factor. Vertical dashed lines mark $a_{\mathrm{c}}$ for each halo. This evolution typically shows many 'spikes' where $f_{\text {sh }}$ sharply increases and then gradually falls off. These peaks correspond to mergers that bring massive satellites inside the main halo (indicated by arrows in the figure), sensibly increasing $f_{\mathrm{sh}}$. Their subsequent drop reflects the mass eroded from the satellite that becomes part of the main halo. Fig. 8 shows that stalled haloes have almost no recent $(a<0.5)$ merger, while accreting haloes undergo many merger 


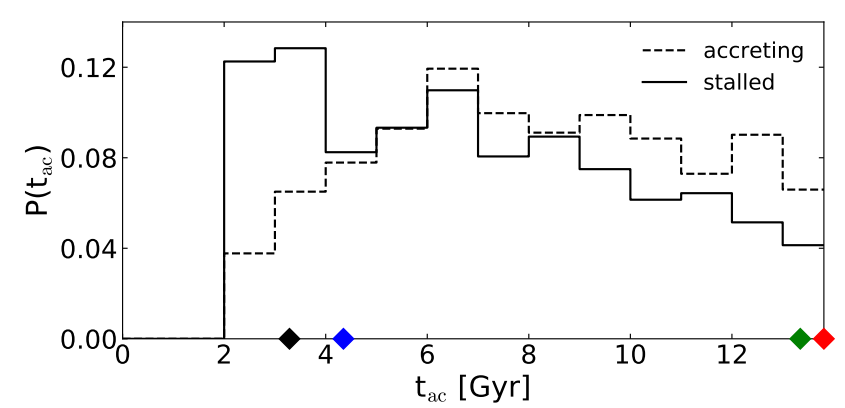

Figure 9. Distribution of accretion times of all the substructures with $M_{\mathrm{sh}} \geq$ $100 m_{\mathrm{DM}}$ that were ever identified within the host. Solid and dashed lines correspond to stalled and accreting haloes, respectively. Diamond symbols show the assembly time of the ZOMG haloes.

events in the same period. This difference reflects their accretion properties, investigated in paper I and II.

The results illustrated above suggest that accreting and stalled haloes host similar subhalo populations at $z=0$ and would be difficult to distinguish observationally. Possible exceptions are: i) the presence of star-forming satellites that only appear in accreting haloes, although in small numbers; ii) the different subhalo mass fractions. In the next section, we investigate the assembly history of the subhalo populations and correlate them with the large-scale environments in which accreting and stalled haloes reside.

\section{EFFECTS OF HALO ASSEMBLY}

In paper I we show that stalled haloes reside within prominent filaments of the cosmic web which distort the matter flow in their surroundings and impact their assembly history and internal dynamics. On the other hand, accreting haloes populate knots of the web and are fed by multiple streams running along thinner filaments. It is then reasonable to expect that their different accretion patterns might impact the infall of satellite and their final configuration.

\subsection{Accretion time}

In Fig. 9 we show the distribution of accretion times $\left(t_{\mathrm{ac}}\right)$ for all surviving and disrupted substructures with $M_{\mathrm{sh}} \geq 100 \mathrm{~m}_{\mathrm{DM}}$. For comparison, we also mark on the $x$-axis the collapse time of the ZOMG haloes using diamond-shaped symbols. Stalled haloes (solid line) show an enhanced accretion of satellites at very early times ( $t_{\mathrm{ac}} \leq 4 \mathrm{Gyr}$ ) with respect to accreting hosts (dashed line) that present a more prominent tail at late epochs $\left(t_{\mathrm{ac}} \geq 9 \mathrm{Gyr}\right)$. The distribution of $t_{\mathrm{ac}}$ is thus consistent with the description of halo collapse discussed in paper I. Interestingly, however, the amount of satellites accreted at late times by accreting haloes is only marginally larger than in stalled hosts.

\subsection{Accretion pattern}

We now investigate if the accretion of satellites follows the same spatial pattern as the DM. The top panels of Fig. 10 show the trajectories (in the rest frame of the host) of all satellites identified at redshift $z=0$ within Amun (left panels) and Supay (right panels) no significant difference is noticeable in Abu and Siris. The colourcoding reflects $M_{\mathrm{sh}}$ as indicated in the bar on the right-hand side.
The black solid and dashed lines highlight the directions of the minor $\left(\hat{\mathbf{e}}_{3}\right)$ and major $\left(\hat{\mathbf{e}}_{1}\right)$ axes of inertia for the Lagrangian patch out of which the halo forms (see paper I for further details). This region is maximally compressed along $\hat{\mathbf{e}}_{1}$ due to tides. For stalled haloes, $\hat{\mathbf{e}}_{3}$ instead coincides with the orientation of the filament they are embedded in. The bottom panels of Fig. 10 show the projection of the orbits on the plane defined by these two directions. For clarity, a few random trajectories are highlighted using coloured lines. The accretion pattern is different in the two classes of haloes. In stalled hosts, substructures first fall in to the filament along $\hat{\mathbf{e}}_{1}$ and then on to the main halo following curved trajectories. This secondary infall can only take place within a small region of the filament immediately surrounding the halo. Beyond this patch, velocities recede from the host along $\hat{\mathbf{e}}_{3}$. It is exactly this configuration that ultimately suppress matter infall and makes the halo stalled. We also note that the gravitational field of the filament bends the trajectories of the infalling satellites with respect to the radial orbits predicted by many idealized collapse models (paper I). On the other hand, accreting haloes have a more isotropic accretion pattern and their infalling substructures reach the host along approximately radial orbits. We conclude that the accretion of satellites and DM are regulated by the same dynamics which is manifestly different in accreting and stalled haloes. Since the distribution and the kinematics of substructures can be constrained with observations, this phenomenon provides us with a chance to distinguish accreting and stalled haloes in the local universe. With this perspective in mind, we explore a number of potential proxies for $z_{\mathrm{c}}$.

\subsection{Subhalo kinematic and anisotropy parameter}

The velocity orientation of substructures at accretion reflects the different infall pattern of accreting and stalled haloes. The solid lines in Fig. 11 show the probability density function (PDF) of the normalized radial velocity component $v_{\mathrm{r}} / v$ at accretion for the ZOMG haloes classified based on their collapse time. In accreting haloes (red), the bulk of the satellites accrete on to their hosts along nearly radial orbits. Conversely, the tangential component is more prominent in stalled haloes (black) where the distribution of $v_{\mathrm{r}} / v$ is uniform. The Kolmogorov-Smirnov (KS) test rules out the null hypothesis that the two samples of subhalo velocities are extracted from the same population at a very high confidence level (the corresponding $p$-value is $p<10^{-300}$ ).

In order to understand if this difference persists with time (and is potentially observable), we analyse the distribution of the satellite velocities at $z=0$ (dashed lines). The PDFs at $z=0$ are much flatter than at accretion as a consequence of the orbit randomization that takes place within the hosts. Nevertheless, accreting haloes still present an excess of satellites on radial orbits (both infalling and outgoing) whilst the substructures of stalled hosts show a small preference for tangential motion $\left(v_{\mathrm{r}} / v \approx 0\right)$. Even in this case it is very unlikely that the samples are drawn from the same underlying population (the $p$-value of the KS test is $2 \times 10^{-12}$ ).

From an observational point of view, determining the state of motion of a population of satellites around a central galaxy is challenging as it requires proper motion measurements. So far this has been possible only for the closest substructures within the Milky Way halo but the Gaia mission and other future space-based facilities might help us extending the measurements to the outer Galaxy and Andromeda (e.g. Wilkinson \& Evans 1999; Kallivayalil et al. 2015). Using 10 satellites with proper motion measurements (the 11 classical satellites with the exception of Sextans), Cautun \& Frenk (2016) conclude that Milky Way satellites show a prominent 


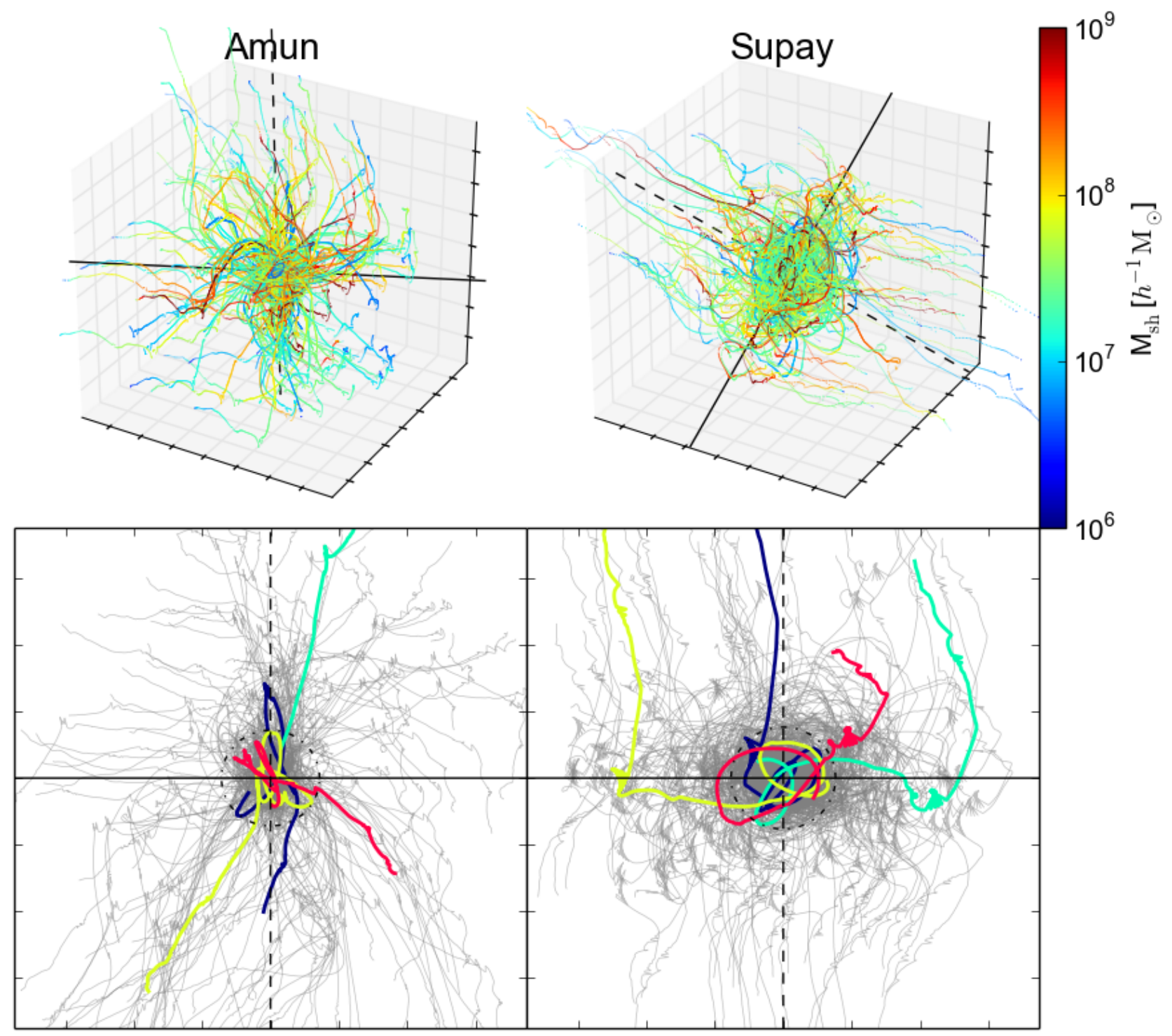

Figure 10. Top: Trajectories of all the substructures identified at $z=0$ in two haloes of the ZOMG suite. The trajectories are in the host rest frame and colourcoded with respect to the subhalo mass. The black solid and dashed lines correspond to the directions of minimum ( $\left.\hat{\mathbf{e}}_{3}\right)$ and maximum $\left(\hat{\mathbf{e}}_{1}\right)$ compression, respectively (see Section 5.2 for details on how they are determined). For Supay, $\hat{\mathbf{e}}_{1}$ corresponds to the direction of the filament it is embedded in. Each panel is $1.5 h^{-1} \mathrm{Mpc}$ wide in each dimension. Bottom: The same as the top panels but projected on the plane defined by $\hat{\mathbf{e}}_{1}$ and $\hat{\mathbf{e}}_{3}$. For visual clarity a random subsample of trajectories have been highlighted using different colours. The dot-dashed circle denotes $R_{\mathrm{h}}$.

excess of tangential orbits which is quite unusual within the $\Lambda \mathrm{CDM}$ paradigm. Interestingly, in paper I, we point out that DM motion in the stalled haloes of the ZOMG suite presents enhanced tangential motions triggered by the gravitational effects of the filaments they are embedded in. In order to connect all these findings, we consider the anisotropy parameter of the satellites

$$
\beta=1-\frac{\sum_{i} v_{\mathrm{tan}, i}^{2}}{2 \sum_{i} v_{\mathrm{rad}, i}^{2}}
$$

where $v_{\mathrm{tan}, i}$ and $v_{\mathrm{rad}, i}$ are the tangential and radial components of the velocity of the $i$-th satellite with respect to the centre of the host halo and the sum runs over the substructures that fulfill a given selection criterion. The value of $\beta$ provides a simple parameterization of the satellite dynamics: $\beta=0$ corresponds to isotropic or- bits while positive and negative values indicate the predominance of tangential and radial orbits, respectively. In Fig. 12, we compare the ZOMG simulations to the results presented in Cautun \& Frenk (2016). The wide vertical shaded band with a dashed line in the middle indicates the measurement and the uncertainty reported for the $10 \mathrm{MW}$ classical satellites. The underlying curve shows the PDF of $\beta$ expected in the $\Lambda$ CDM scenario. This has been extracted from an $N$-body simulation whose haloes have been populated with galaxies using a semi-analytical model. To build the PDF, Cautun \& Frenk (2016) first select MW-type haloes and then consider only the 10 satellites with the largest stellar mass for each halo. Note that only 2.9 per cent of the $\Lambda \mathrm{CDM}$ haloes are associated with a more extreme value of $\beta$ than the MW. The values we obtain for the four ZOMG haloes are shown with vertical solid lines surrounded 


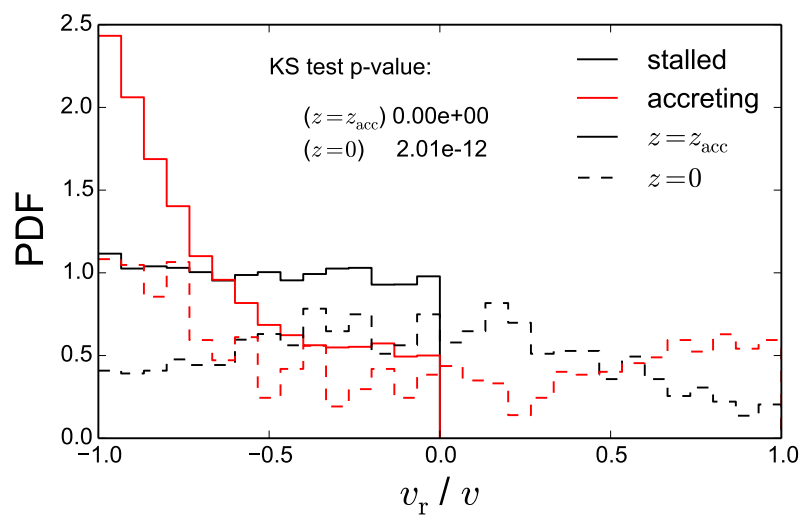

Figure 11. Probability density function (PDF) of the (normalized) radial velocity at the time of accretion (solid lines) and at $z=0$ (dashed lines) for substructures hosted by stalled (black lines) and accreting (red lines) haloes. Distributions referring to the same time are compared using a KolmogorovSmirnov test and the resulting $p$-value is reported.

by a shaded band which indicates the corresponding jacknife errors. To explore the dependence of $\beta$ on the satellite sample we repeat the measurements using four different selection criteria: the ten satellites with the largest stellar mass ('brightest', top), all the subhaloes containing at least one stellar particle ('all bright', upper centre), the ten most massive satellites in the hydrodynamic simulations ('most massive', lower centre) and in the DM-only runs (bottom). It is worth noticing that, in the hydrodynamic simulations and when we select the 10 most massive subhaloes (either based on the stellar or the total mass), Supay has a more extreme value of $\beta$ than it is measured for the MW.

Although the precise values of $\beta$ vary significantly with the sample, stalled (accreting) haloes invariably show negative (positive) values of $\beta$. This is a consequence of the large difference in the radial velocities at accretion time (see Fig. 11), which is preserved until $z=0$. Additionally, substructures in accreting haloes tend to have larger $t_{\mathrm{ac}}$ than in stalled hosts (see Fig. 9, we check that this holds true for all the selection criteria employed) that are therefore more affected by orbit randomization.

The results presented in this section suggest a way forward to link observable properties of satellites to the assembly time of their host DM halo. They represent an evidence that satellite dynamics is strongly affected by the environment, which in turn determines the collapse time of the halo (paper I). Hence, if the proper motion of sufficiently numerous satellites can be measured (e.g. in the local universe), stalled and accreting DM haloes can in principle be distinguished. Additionally, in paper II we show that stalled haloes are found to host central galaxies with thicker stellar discs and older stellar populations with respect to accreting haloes. By considering a large sample of haloes, a dedicated numerical effort could then determine a precise relation between all these characteristics and $t_{\mathrm{c}}$.

Based on our results, it is tempting to classify the MW halo as a stalled structure. In fact, the anisotropy parameter of its bright satellites is significantly negative. Consistently, the stellar disc of the Galaxy is found to be relatively thick and old (Gilmore \& Reid 1983; Dalcanton \& Bernstein 2002). Something to bear in mind is that, contrary to naive expectations, plenty of gas can still accrete on to the central galaxy of a stalled halo and sustain regular star formation (see paper II).

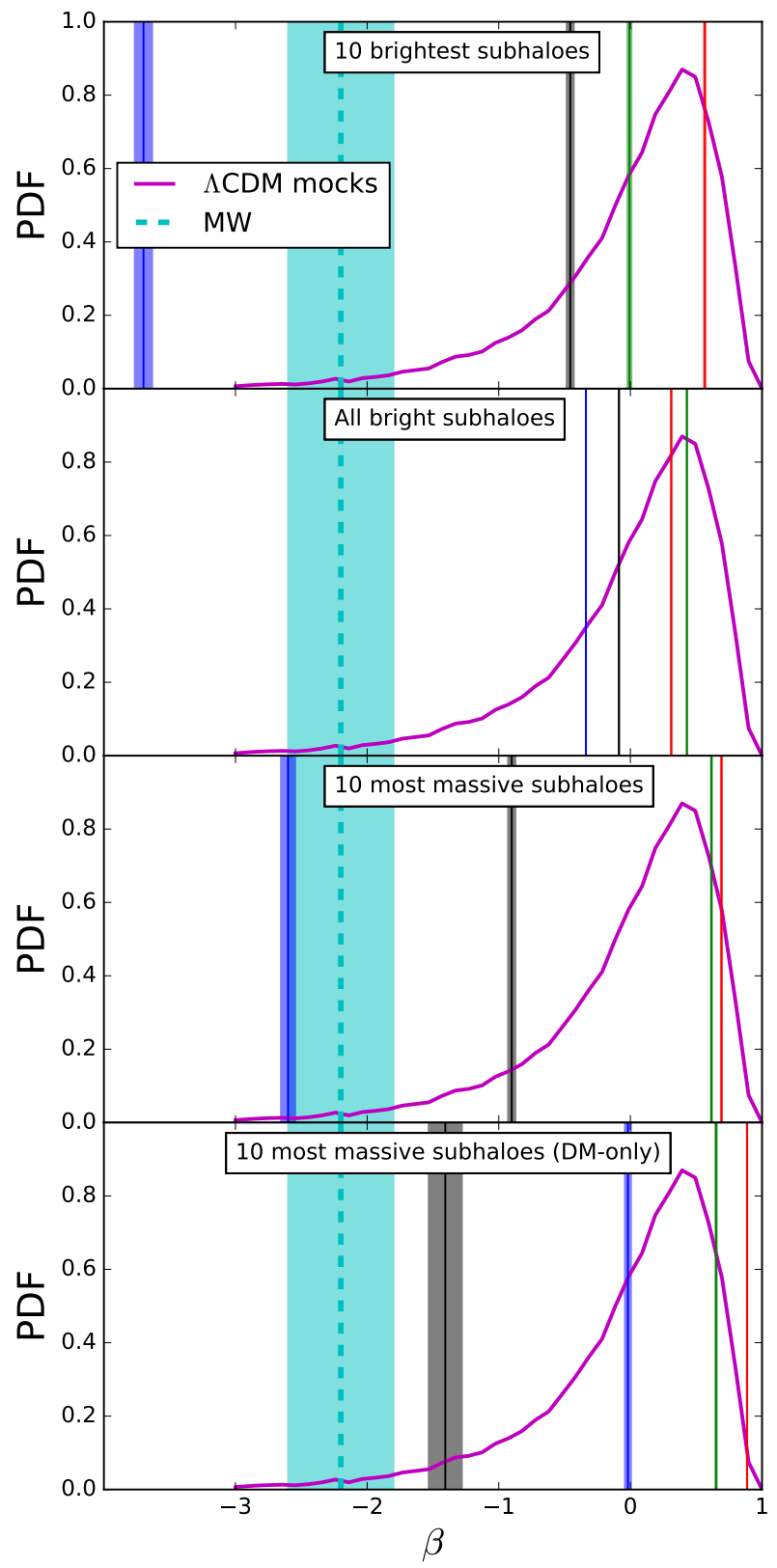

Figure 12. Anisotropy parameter $\beta$ computed for our halo sample (solid vertical lines, the shaded region around indicates the bootstrap error). The dashed vertical cyan line (and its shading) shows the results for the MW classical satellites from Cautun \& Frenk (2016), while the magenta profile shows the probability distribution function computed by the same authors using a semi-analytical model applied to MW-type DM-haloes. The four panels show different subsets of satellites used to compute the anisotropy parameter, namely (from top to bottom) the ten with largest stellar mass, all bright satellites with at least one stellar particle, the ten most massive in the hydrodynamical runs and in the DM-only ones.

The dependence of the anisotropy parameter on $t_{\mathrm{c}}$ might help shedding new light on another intriguing observational finding. Investigating the satellite kinematics in the Sloan Digital Sky Survey, Wojtak \& Mamon (2013) infer that the satellites of red galaxies tend to have a positive $\beta$. Taken at face value, this result might suggest that red central galaxies are preferentially hosted by accreting 
Table 2. The spatial flatness of a given set of substructures can be determined by computing the ratio between the lengths of their minor and major axes of inertia $(c / a)$. Below we report the values of $c / a$ obtained applying different selection criteria for the satellites. Namely, MM refers to the ten most massive satellites, $\mathrm{MB}$ to the ten brightest, $\mathrm{AB}$ to all the substructures with at least one stellar particle, and DM to the ten subhaloes with the largest $M_{\mathrm{sh}}$ in the DM-only run. We repeat each calculation three times, using different weighting schemes: uniform, $w=r^{-1}$ and $w=r^{-2}$, where $r$ denotes the distance of a satellite from the centre of its host halo.

\begin{tabular}{lccc|ccc} 
& $w=1$ & $w=r^{-1}$ & $w=r^{-2}$ & $w=1$ & $w=r^{-1}$ & $w=r^{-2}$ \\
\cline { 2 - 7 } & \multicolumn{3}{c}{ Abu } & & \multicolumn{3}{c}{ Supay } \\
MM & 0.09 & 0.19 & 0.33 & 0.52 & 0.61 & 0.33 \\
MB & 0.17 & 0.26 & 0.32 & 0.14 & 0.18 & 0.20 \\
AB & 0.43 & 0.49 & 0.53 & 0.44 & 0.46 & 0.44 \\
DM & 0.12 & 0.20 & 0.33 & 0.12 & 0.19 & 0.22 \\
\cline { 2 - 7 } & \multicolumn{3}{c}{ Amun } & \multicolumn{3}{c}{ Siris } \\
MyM & 0.12 & 0.15 & 0.14 & 0.48 & 0.45 & 0.38 \\
MB & 0.06 & 0.10 & 0.17 & 0.44 & 0.52 & 0.40 \\
AB & 0.25 & 0.29 & 0.34 & 0.45 & 0.39 & 0.33 \\
DM & 0.14 & 0.18 & 0.20 & 0.30 & 0.37 & 0.41 \\
\cline { 2 - 7 } & & & & & &
\end{tabular}

haloes. In the ZOMG simulations, no obvious correlation has been found between the central galaxy type and the halo accretion history (paper II). However, the ZOMG suite is too small to detect subtle statistical trends and substantially larger numerical samples are required to draw strong conclusions regarding the interpretation of the observational data.

\subsection{Satellite configuration}

The brightest substructures of the MW lie within a flattened region which is almost perpendicular to the Galactic disc (Kunkel \& Demers 1976; Lynden-Bell 1976). Proper motion measurements of the classical satellites suggest that most of them orbit within this 'plane' (Kroupa et al. 2005). Likewise, nearly half of Andromeda's satellites form a thin planar structure (Ibata et al. 2013). The existence of similar features has also been reported for M81 (Chiboucas et al. 2013) and Centaurus A (Tully et al. 2015). In addition, the dwarf galaxies within the NGC 3109 association form a filamentary structure which is well ordered in phase space (Bellazzini et al. 2013). Within the $\Lambda$ CDM paradigm, a few mechanisms have been invoked to explain the presence of these dynamically coherent features. Among these, for instance, are the hypothesis that substructures accrete on to the host while they are part of clustered groups (Li \& Helmi 2008; D’Onghia \& Lake 2008) or that cosmic filaments imprint a preferential direction for the infall of satellites (Libeskind et al. 2011; Lovell et al. 2011). It is difficult to conciliate this scenario with the observations in a quantitative way (e.g. Pawlowski et al. 2012). As an alternative, it has been proposed that the planes of satellites in the MW and in Andromeda might originate from the tidal tail of a violent galaxy interaction that took place long ago in the Local Group (e.g. Hammer et al. 2013, and references therein). Although a consensus has yet to be reached on this issue, it is anyway interesting to further explore the implications of the standard cosmological model for the spatial distribution of the satellites. For instance, the different accretion modes of stalled and accreting haloes could play a key role in shaping more or less flattened collections of substructures at $z=0$. Therefore, we investigate the planarity of the satellite distributions in the ZOMG haloes.
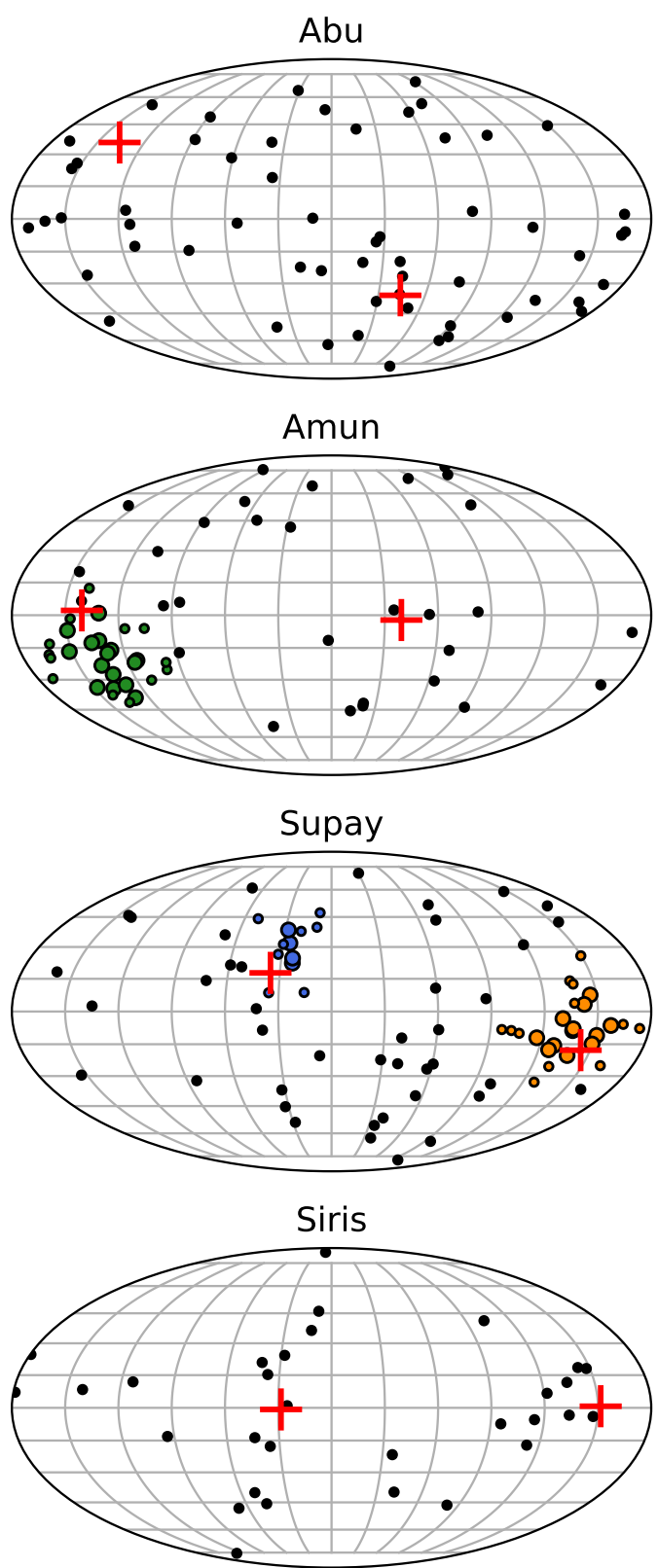

Figure 13. Mollweide maps showing the direction of the orbital angular momentum for our simulated satellite galaxies with $M_{*}>10^{5} h^{-1} \mathrm{M}_{\odot}$. Each $L$-cluster identified by DBSCAN is highlighted with a different colour while black symbols denote unclustered satellites. The red crosses indicate the smallest principal axis of the ToI for the 'all bright' subsample evaluated with uniform weights.

\subsubsection{Spatial distribution}

We first focus on the satellite positions at $z=0$ for the four subsamples we already used in Section 5.3 although a more general analysis will be presented in Section 5.4.2. We compute the tensor of inertia (ToI) of the substructures employing three different weighting schemes (uniform, $r^{-1}$ and $r^{-2}$ where $r$ is the radial distance from the halo centre). We compute the eigenvalues of the ToI and denote by $a \geq b \geq c$ their square roots. The degree of planarity of the satellite distribution is quantified using the ratio $c / a$. Our re- 

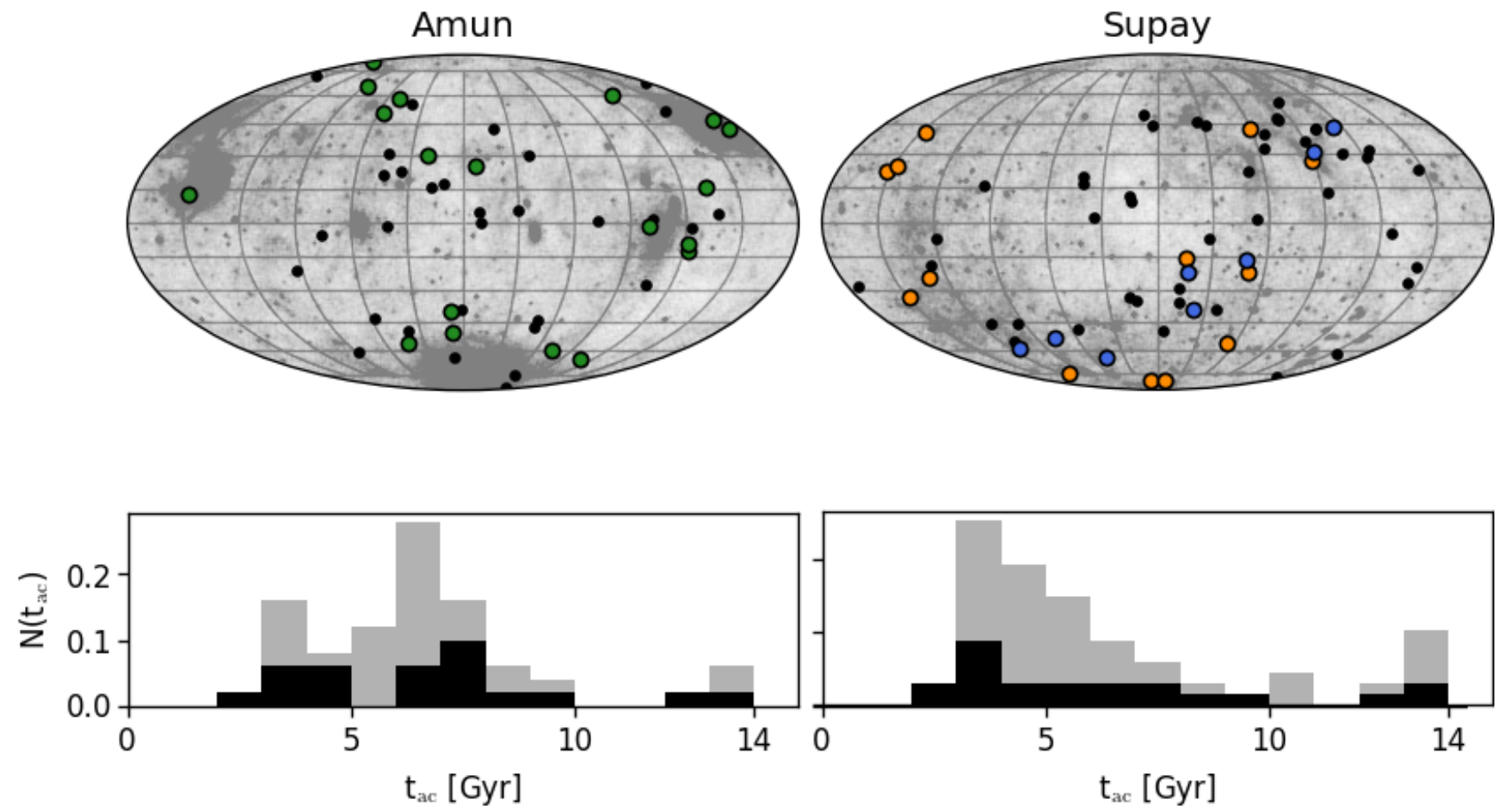

Figure 14. Top: Mollweide map of the angular position at accretion time of the satellites with $M_{*}>10^{5} h^{-1} \mathrm{M}_{\odot}$ in Supay and Amun (circles, colour-coded as in Fig. 13). In the background, we show the matter distribution at $R_{\mathrm{h}}$ evaluated at $z=0$ (darker regions correspond to higher surface densities). The few thin filaments that converge in Amun are easily seen whereas the filament embedding Supay is not noticeable because it is much thicker than $R_{\mathrm{h}}$. Bottom: Probability distribution of $t_{\mathrm{ac}}$ for all satellites with $M_{*}>10^{5} h^{-1} \mathrm{M}_{\odot}$ (gray) and for the satellites in the $L$-clusters (black). For Supay both $L$-clusters have been combined together.

sults are presented in Table 2. Although the measurements sensibly depend on the sample and the weighting scheme, the brightest (or most massive) satellites in accreting haloes tend to lie within flatter spatial configurations with respect to those in stalled hosts. However, given the variability of the results, it is difficult to draw solid conclusions based on our small sample. In many cases, the values of $c / a$ extracted from the simulations are comparable with the estimates $^{2}$ found for the MW which range between 0.18 (Metz et al. 2008) and 0.3 (Libeskind et al. 2005).

We now test whether the flattened satellite distributions in the ZOMG haloes are dynamically stable. If this is the case, the angular momenta of the satellites should be well aligned (or anti-aligned) with the normal, $\hat{\mathbf{n}}$, to the 'plane'. We therefore compute the angle $\theta_{i}$ between the orbital angular momentum of a substructure and the smallest principal axis of the ToI (a proxy for $\hat{\mathbf{n}}$ ). In many cases, only a few of the satellites that have been used to identify $\hat{\mathbf{n}}$ actually have $\left|\cos \left(\theta_{i}\right)\right| \sim 1$. This suggests that the planar structures determined with the ToI are short lived and probably due to chance alignments. In some haloes, however, 60-70 per cent of the selected satellites have $\left|\cos \left(\theta_{i}\right)\right|>0.75$ and can orbit in the flattened struc-

2 Maji et al. (2017a,b) find that estimates of $c / a$ are not stable and argue against the existence of a plane of satellites in the MW. Pawlowski et al. (2017) strongly criticize this claim and emphasize the lack of a proper statistical analysis to reach it. Our work consists of a theoretical investigation on the impact of the assembly history on the substructure properties. Drawing conclusions about the MW satellites is outside the scope of this paper and, in any case, our simulation suite would be too small to conduct such an investigation. tures for longer times. This happens, for instance, for both the accreting haloes when we consider the most massive substructures (based on the KS test the difference with the stalled haloes is significant at 99 per cent confidence level) and for Abu and Supay when we select the brightest satellites. Interestingly, $c / a$ is rather low in these cases.

\subsubsection{Clustering of angular momenta}

To generalize the results presented in the previous section, we now look for groups of substructures with aligned orbital angular momenta without pre-selecting the satellites based on their structural properties, as we did before to compare with previous work. We only require the stellar mass to be $M_{*}>10^{5} h^{-1} \mathrm{M}_{\odot}$ to approximately match the observational limits. Coherently rotating groups are identified by applying a clustering algorithm to the directions of the orbital angular momenta. We use the method named DensityBased Spatial Clustering of Applications with Noise (DBSCAN, Ester et al. 1996) and make sure that our analysis does not depend on its tunable parameters. The resulting $L$-clusters are shown in Fig. 13 (coloured filled circles) together with all the unclustered satellites (black circles). The angular momenta of the substructures in $\mathrm{Abu}$ and Siris are consistent with a random distribution. On the other hand, Amun shows a prominent $L$-cluster composed of 25 objects and Supay presents two of them containing 9 and 21 satellites. Interestingly, these two groups orbit in opposite directions in the plane perpendicular to the vector $\hat{\mathbf{n}}$ identified for the 'all bright' subsample (red crosses). It is worth noticing that Amun and Supay are the only two haloes in our simulations that host a grand-design 


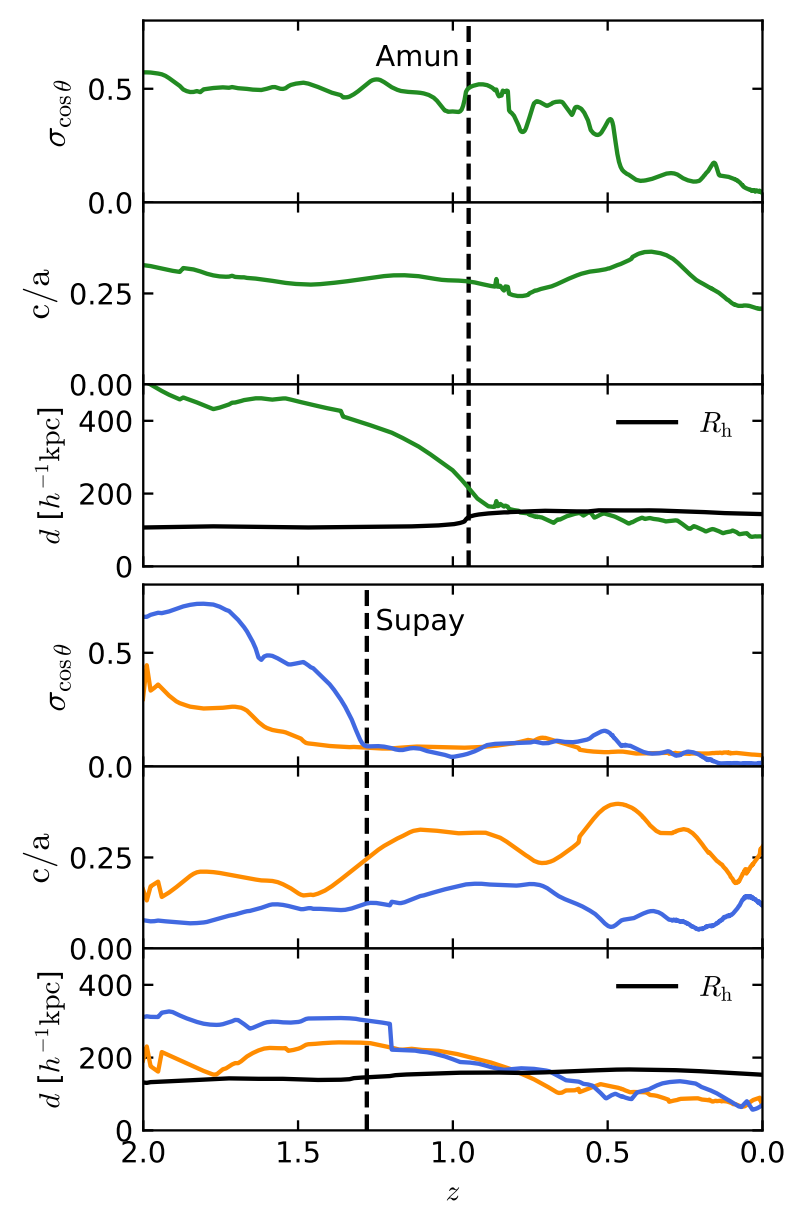

Figure 15. The degree of alignment of the orbital angular momenta $\left(\sigma_{\cos \theta}\right.$, top), the level of planarity $(c / a$, centre) and the median distance from the host centre $(d$, bottom) are plotted as a function of time for the satellites that at $z=0$ form the $L$-clusters in Supay and Amun. Each $L$-cluster is denoted by the colour used in Fig. 13. The vertical dashed lines indicate the redshift of the last major merger for the host haloes. For each snapnshot we only consider substructures containing at least $20 \mathrm{DM}$ particles.

spiral galaxy (paper II). Hence, it is not inconceivable that related physical mechanisms lie at the origin of both the galactic disc and the satellite plane. We have checked that the same $L$-clusters are also present in the DM-only simulations suggesting that baryons do not play a key role in their formation process. Despite the ZOMG sample is only formed of four central galaxies, two of them are surrounded by evident planes of satellites which are dynamically coherent. Our results thus suggest that the occurrence of these structures in the $\Lambda \mathrm{CDM}$ paradigm is not unlikely.

In the remainder of this section, we investigate the possible origin of the planar satellite configurations found in Amun and Supay. In the top panels of Fig. 14 we correlate the satellite position at accretion time (circles) with the matter distribution at $R_{\mathrm{h}}$ (gray background). Substructures are colour-coded as in Fig. 13. Although satellites tend to accrete preferentially from high-density regions, the members of each $L$-cluster do not come from the same direction. Moreover, the distribution of their accretion times fairly traces that of all the luminous satellites (bottom panels in Fig. 14). These results do not support filamentary and/or clustered accretion as an explanation for the formation of the planar structures.
In order to understand if the $L$-clusters are short- or long-lived, we study their time evolution in Fig. 15. We characterize their degree of coherence using the rms value $\left(\sigma_{\cos \theta}\right)$ of $\cos \theta$ defined with respect to their mean orbital angular momentum $\left(\sigma_{\cos \theta}=3^{-1 / 2} \simeq\right.$ 0.577 for a random distribution while $\sigma_{\cos \theta}=0$ for a perfectly coherent cluster). Simultaneously, we use $c / a$ (employing uniform weights for the ToI) to describe their flatness and compute the median distance $(d)$ of the cluster members from the host. For both galaxies, $\sigma_{\cos \theta}$ decreases significantly with time while $c / a$ stays approximately constant. Note that the redshift range covered in Fig. 15 extends well beyond the accretion time of the substructures. As a reference, we indicate the time of the last major merger (determined from the mass accretion history) using a vertical dashed line. The $L$-clusters in Supay are already identifiable at early times (even for $t<t_{\mathrm{c}}$, at least for the smaller one) when most of the substructures are still beyond $R_{\mathrm{h}}$. This is also noticeable in Fig. 14 where the angular positions at accretion time of most cluster members lie within a very flattened region. Based on timing arguments, the formation of the $L$-clusters in Supay appears to be connected with the final stages of assembly of the host halo. On the other hand, the dynamically coherent group in Amun rapidly forms at much later times $(z \sim 0.4)$, when more than 50 per cent of its members are already within the host halo. This might possibly reflect the delayed evolution of accreting haloes with respect to stalled ones.

\section{CONCLUSIONS}

We have exploited the high mass and temporal resolution of the ZOMG simulation suite (paper I, paper II) to study the substructure evolution of four haloes with masses of a few $\times 10^{11} h^{-1} \mathrm{M}_{\odot}$ at $z=0$. Abundance matching shows that haloes of this size have most efficiently converted baryons into stars and host $L_{*}$ galaxies at the present time. We have characterized the assembly history of the haloes in terms of their collapse redshift, $z_{\mathrm{c}}$, defined as the epoch at which the physical volume enclosing the halo material first becomes stable. Galaxy-sized DM haloes identified at $z=0$ show a broad distribution of $z_{\mathrm{c}}$ (see Fig. 2 in paper I). In paper I, we have shown that $z_{\mathrm{c}}$ correlates with the cosmic environment surrounding the halo: stalled haloes are embedded within prominent filaments of the cosmic web that inhibit further infall of matter while accreting haloes are located at the knots of the web and are fed by a number of thinner filamentary structures. In order to study which properties of the haloes (and of the galaxies within them) depend on $z_{\mathfrak{c}}$, we have selected targets for zoom hydrodynamic simulations by sampling the tails of this distribution. We thus ended up considering two 'accreting' haloes $\left(z_{\mathrm{c}} \lesssim 0\right)$ named Abu and Amun and two 'stalled' haloes $\left(z_{\mathrm{c}} \gtrsim 1\right)$ dubbed Siris and Supay.

Our analysis reveals that many properties of the substructures are insensitive to the assembly history of the host halo. We list these features below.

(i) At $z=0$, more than 80 per cent of the surviving substructures do not contain stars (consistently with the effect of reionization) and more than 99 per cent are stripped off of the their entire gas content (in agreement with observations of dwarf spheroidal satellites of the Milky Way).

(ii) Based on our feedback scheme, roughly half of the gas brought in the main halo by satellites is subsequently ejected and remains outside $R_{\mathrm{h}}$ until $z=0$.

(iii) The fraction of baryonic mass in the satellites (identified at $z=2$ ) that ends up in the disc of the central galaxy at $z=0$ correlates with the disc size and $M_{\mathrm{sh}}$. This reflects both the strength 
of the gravitational field generated by the disc (Garrison-Kimmel et al. 2017) and the increased ability to retain a gas reservoir that can be then deposited in the central galaxy by the most massive substructures.

(iv) The first apocentre of nearly 40 per cent of the satellites is located beyond the 'splashback radius' of the host halo identified as a sudden steepening of the mass density profile. This indicates that further work is needed to connect $R_{\mathrm{spl}}$ with the orbits of recently accreted material.

(v) The evolved (i.e. at $z=0$ ) and unevolved (i.e. at accretion time) mass functions, the radial distribution, the spread of the stellar mass fraction and of the velocity dispersion of the substructures are insensitive to the collapse time of the halo.

(vi) Based on their sSFR at $z=0$, all the central and satellite galaxies are classified as 'quenched' according to the criterion of Weinmann et al. (2006). The ZOMG sample therefore shows perfect conformity between the SF properties of primary and satellite galaxies and confirms the trend previously found for larger halo masses.

(vii) Two of our resimulated haloes (Amun and Supay) contain large clusters of satellites with aligned orbital angular momenta that form a flattened structure in space. The very same features are present in the zoom $N$-body simulations of the haloes thus suggesting that baryonic physics does not play a major role in their formation. Contrary to other studies, we found that these clusters do not collect satellites that fell in along a specific direction or as a coherent group. Intriguingly, Amun and Supay are the only haloes in our sample that host a grand-design spiral galaxy at their centre thus suggesting a possible connection between the physics of disc formation and the assembly of planar configurations of satellites.

On the other hand, additional properties of the substructures clearly depend on the assembly history of the host halo. Most of them are related to the spatial and temporal pattern of satellite accretions that directly reflect the different cosmic environments hosting accreting and stalled haloes. They can be summarized as follows.

(viii) The fraction of halo mass locked in substructures at $z=$ 0 is substantially larger in accreting haloes. Concurrently, stalled (accreting) haloes accrete a larger fraction of satellites at early (late) times.

(ix) The epoch at which the planar structures of satellites are formed (see item (vi) above) seems to be connected with the assembly time of the host haloes.

(x) Substructures fall in towards accreting haloes following nearly radial trajectories. On the contrary, satellites initially orbit the filament that embeds the stalled haloes before falling on to their hosts. For this reason, they have a large tangential velocity component at accretion time.

(xi) Although maximal at infall, the different balance between the radial and tangential components of the satellite velocities in accreting and stalled haloes is clearly noticeable also at later times. For instance, the velocity anisotropy parameter of the satellites at $z=0$ is positive for accreting haloes and negative for stalled haloes. This finding parallels the result found in paper I for the DM particles and provides a tool to determine the formation time of a halo based on the kinematic properties of its satellite galaxies. Cautun \& Frenk (2016) have recently measured a strong tangential excess for the classical MW satellites corresponding to an anisotropy parameter of $\beta=-2.2 \pm 0.4$. It is thus tempting to tentatively categorize the MW halo as stalled. Further support to this conjecture comes from the thickness and age of the stellar disc (paper II).
Future work will aim to extend the approach presented in this work to a larger sample of haloes covering a wider range of halo masses and collapse times.

\section{ACKNOWLEDGEMENTS}

We thank the anonymous referee for suggesting to discuss galactic conformity, Marius Cautun for useful discussions, Philip Mansfield for help with the SHELLFISH code and Volker Springel for making PGADGET-3 available to us. This work is carried out within the SFB 956 'The Conditions and Impact of Star Formation', sub-project C4, and the Transregio 33 'The Dark Universe' projects funded by the Deutsche Forschungsgemeinschaft (DFG). MB thanks the Bonn-Cologne Graduate School for Physics and Astronomy for support. The results presented were achieved employing computing resources (Cartesius) at SURF/SARA, The Netherlands as part of the PRACE-3IP project (FP7 RI-312763). We are thankful to the community developing and maintaining software packages extensively used in our work, namely: Matplotlib (Hunter 2007), NumPy (Walt et al. 2011), scikit-learn (Pedregosa et al. 2011), SciPy (Jones et al. 2001).

\section{REFERENCES}

Adhikari S., Dalal N., Chamberlain R. T., 2014, J. Cosmology Astropart. Phys., 11, 019

Ahmed S. H., Brooks A. M., Christensen C. R., 2017, MNRAS, 466, 3119 Baxter E., et al., 2017, ApJ, 841, 18

Bellazzini M., Oosterloo T., Fraternali F., Beccari G., 2013, A\&A, 559, L11 Benítez-Llambay A., Navarro J. F., Abadi M. G., Gottlöber S., Yepes G., Hoffman Y., Steinmetz M., 2016, MNRAS, 456, 1185

Binney J., Tremaine S., 2008, Galactic Dynamics: Second Edition. Princeton University Press

Borzyszkowski M., Ludlow A. D., Porciani C., 2014, MNRAS, 445, 4124

Borzyszkowski M., Porciani C., Romano-Díaz E., Garaldi E., 2017, MNRAS, 469, 594

Buck T., Macciò A. V., Dutton A. A., 2015, ApJ, 809, 49

Bullock J. S., 2010, preprint, (arXiv: 1009.4505)

Cautun M., Frenk C. S., 2016, preprint, (arXiv: 1612.01529)

Cautun M., Hellwing W. A., van de Weygaert R., Frenk C. S., Jones B. J. T., Sawala T., 2014, MNRAS, 445, 1820

Chiboucas K., Jacobs B. A., Tully R. B., Karachentsev I. D., 2013, AJ, 146, 126

Chua K. T. E., Pillepich A., Rodriguez-Gomez V., Vogelsberger M., Bird S., Hernquist L., 2016, preprint, (arXiv: 1611.07991)

D’Onghia E., Lake G., 2008, ApJ, 686, L61

Dalal N., White M., Bond J. R., Shirokov A., 2008, ApJ, 687, 12

Dalcanton J. J., Bernstein R. A., 2002, AJ, 124, 1328

Di Cintio A., Knebe A., Libeskind N. I., Brook C., Yepes G., Gottlöber S., Hoffman Y., 2013, MNRAS, 431, 1220

Diemer B., 2017, ApJS, 231, 5

Diemer B., Kravtsov A. V., 2014, ApJ, 789, 1

Dolag K., Borgani S., Murante G., Springel V., 2009, MNRAS, 399, 497

Doroshkevich A. G., Zel'dovich Y. B., Novikov I. D., 1967, Soviet Ast., 11, 233

Dutton A. A., 2012, MNRAS, 424, 3123

Ester M., Kriegel H.-P., Sander J., Xu X., et al., 1996, in Kdd. pp 226-231

Gao L., White S. D. M., 2007, MNRAS, 377, L5

Gao L., Springel V., White S. D. M., 2005, MNRAS, 363, L66

Gao L., Frenk C. S., Boylan-Kolchin M., Jenkins A., Springel V., White S. D. M., 2011, MNRAS, 410, 2309

Gao L., Navarro J. F., Frenk C. S., Jenkins A., Springel V., White S. D. M., 2012, MNRAS, 425, 2169

Garrison-Kimmel S., et al., 2017, preprint, (arXiv: 1701.03792) 
Ghigna S., Moore B., Governato F., Lake G., Quinn T., Stadel J., 2000, ApJ, 544,616

Gill S. P. D., Knebe A., Gibson B. K., 2004, MNRAS, 351, 399

Gilmore G., Reid N., 1983, MNRAS, 202, 1025

Giocoli C., Tormen G., van den Bosch F. C., 2008, MNRAS, 386, 2135

Gnedin N. Y., 2000, ApJ, 542, 535

González R. E., Padilla N. D., 2016, ApJ, 829, 58

Grcevich J., Putman M. E., 2009, ApJ, 696, 385

Haardt F., Madau P., 2001, in Neumann D. M., Tran J. T. V., eds, Clusters of Galaxies and the High Redshift Universe Observed in X-rays. (arXiv:astro-ph/0106018)

Hahn O., Abel T., 2011, MNRAS, 415, 2101

Hahn O., Porciani C., Dekel A., Carollo C. M., 2009, MNRAS, 398, 1742

Hammer F., Yang Y., Fouquet S., Pawlowski M. S., Kroupa P., Puech M., Flores H., Wang J., 2013, MNRAS, 431, 3543

Han J., Cole S., Frenk C. S., Jing Y., 2016, MNRAS, 457, 1208

Harker G., Cole S., Helly J., Frenk C., Jenkins A., 2006, MNRAS, 367, 1039

Hellwing W. A., Frenk C. S., Cautun M., Bose S., Helly J., Jenkins A., Sawala T., Cytowski M., 2016, MNRAS, 457, 3492

Helmi A., White S. D. M., 1999, MNRAS, 307, 495

Hirschmann M., Naab T., Somerville R. S., Burkert A., Oser L., 2012, MNRAS, 419, 3200

Hunter J. D., 2007, Computing In Science \& Engineering, 9, 90

Ibata R. A., et al., 2013, Nature, 493, 62

Ishiyama T., et al., 2013, ApJ, 767, 146

Jones E., Oliphant T., Peterson P., et al., 2001, SciPy: Open source scientific tools for Python, http: //www. scipy.org/

Kallivayalil N., et al., 2015, preprint, (arXiv: 1503.01785)

Kauffmann G., Li C., Zhang W., Weinmann S., 2013, MNRAS, 430, 1447

Klypin A., Gottlöber S., Kravtsov A. V., Khokhlov A. M., 1999, ApJ, 516, 530

Knobel C., Lilly S. J., Woo J., Kovač K., 2015, ApJ, 800, 24

Knollmann S. R., Knebe A., 2009, ApJS, 182, 608

Kravtsov A., 2010, Advances in Astronomy, 2010, 281913

Kroupa P., Theis C., Boily C. M., 2005, A\&A, 431, 517

Kunkel W. E., Demers S., 1976, in Dickens R. J., Perry J. E., Smith F. G., King I. R., eds, Royal Greenwich Observatory Bulletins Vol. 182, The Galaxy and the Local Group. p. 241

Lelli F., McGaugh S. S., Schombert J. M., 2016, ApJ, 816, L14

Li Y.-S., Helmi A., 2008, MNRAS, 385, 1365

Libeskind N. I., Frenk C. S., Cole S., Helly J. C., Jenkins A., Navarro J. F., Power C., 2005, MNRAS, 363, 146

Libeskind N. I., Cole S., Frenk C. S., Okamoto T., Jenkins A., 2007, MNRAS, 374, 16

Libeskind N. I., Knebe A., Hoffman Y., Gottlöber S., Yepes G., Steinmetz M., 2011, MNRAS, 411, 1525

Libeskind N. I., Knebe A., Hoffman Y., Gottlöber S., 2014, MNRAS, 443, 1274

Libeskind N. I., Hoffman Y., Tully R. B., Courtois H. M., Pomarède D., Gottlöber S., Steinmetz M., 2015, MNRAS, 452, 1052

Liu L., Gerke B. F., Wechsler R. H., Behroozi P. S., Busha M. T., 2011, ApJ, 733, 62

Lovell M. R., Eke V. R., Frenk C. S., Jenkins A., 2011, MNRAS, 413, 3013

Ludlow A. D., Navarro J. F., Springel V., Jenkins A., Frenk C. S., Helmi A., 2009, ApJ, 692, 931

Lynden-Bell D., 1976, MNRAS, 174, 695

Maji M., Zhu Q., Marinacci F., Li Y., 2017a, preprint, (arXiv: 1702.00485)

Maji M., Zhu Q., Marinacci F., Li Y., 2017b, preprint, (arXiv: 1702.00497)

Mansfield P., Kravtsov A. V., Diemer B., 2016, preprint, (arXiv: 1612.01531)

Mayer L., 2010, Highlights of Astronomy, 15, 193

McConnachie A. W., 2012, AJ, 144, 4

McGaugh S. S., 2012, AJ, 143, 40

McGaugh S. S., Schombert J. M., Bothun G. D., de Blok W. J. G., 2000, ApJ, 533, L99
Metz M., Kroupa P., Libeskind N. I., 2008, ApJ, 680, 287

Mo H., van den Bosch F. C., White S., 2010, Galaxy Formation and Evolution

Mohayaee R., Shandarin S. F., 2006, MNRAS, 366, 1217

Moore B., Katz N., Lake G., 1996, ApJ, 457, 455

Moore B., Ghigna S., Governato F., Lake G., Quinn T., Stadel J., Tozzi P., 1999, ApJ, 524, L19

More S., Diemer B., Kravtsov A. V., 2015, ApJ, 810, 36

More S., et al., 2016, ApJ, 825, 39

Nagai D., Kravtsov A. V., 2005, ApJ, 618, 557

Navarro J. F., Frenk C. S., White S. D. M., 1997, ApJ, 490, 493

Okabe N., Futamase T., Kajisawa M., Kuroshima R., 2014, ApJ, 784, 90

Okamoto T., Gao L., Theuns T., 2008, MNRAS, 390, 920

Okamoto T., Frenk C. S., Jenkins A., Theuns T., 2010, MNRAS, 406, 208

Paranjape A., Kovač K., Hartley W. G., Pahwa I., 2015, MNRAS, 454, 3030

Parry O. H., Eke V. R., Frenk C. S., Okamoto T., 2012, MNRAS, 419, 3304

Pawlowski M. S., Kroupa P., Angus G., de Boer K. S., Famaey B., Hensler G., 2012, MNRAS, 424, 80

Pawlowski M. S., et al., 2017, preprint, (arXiv: 1702.06143)

Pedregosa F., et al., 2011, Journal of Machine Learning Research, 12, 2825

Planck Collaboration et al., 2014, A\&A, 571, A16

Rees M. J., 1986, MNRAS, 218, 25P

Romano-Díaz E., Shlosman I., Heller C., Hoffman Y., 2010, ApJ, 716, 1095

Romano-Díaz E., Garaldi E., Borzyszkowski M., Porciani C., 2017, MNRAS, 469, 1809

Sales L. V., et al., 2017, MNRAS, 464, 2419

Sawala T., Frenk C. S., Crain R. A., Jenkins A., Schaye J., Theuns T., Zavala J., 2013, MNRAS, 431, 1366

Sawala T., Pihajoki P., Johansson P. H., Frenk C. S., Navarro J. F., Oman K. A., White S. D. M., 2017, MNRAS, 467, 4383

Scannapieco C., et al., 2012, MNRAS, 423, 1726

Schewtschenko J. A., Macciò A. V., 2011, MNRAS, 413, 878

Shao S., Cautun M., Frenk C. S., Gao L., Crain R. A., Schaller M., Schaye J., Theuns T., 2016, MNRAS, 460, 3772

Sorce J. G., Guo Q., 2016, MNRAS, 458, 2667

Spekkens K., Urbancic N., Mason B. S., Willman B., Aguirre J. E., 2014, ApJ, 795, L5

Springel V., 2005, MNRAS, 364, 1105

Springel V., Hernquist L., 2003, MNRAS, 339, 289

Springel V., et al., 2008, MNRAS, 391, 1685

Tormen G., Diaferio A., Syer D., 1998, MNRAS, 299, 728

Tormen G., Moscardini L., Yoshida N., 2004, MNRAS, 350, 1397

Tully R. B., Fisher J. R., 1977, A\&A, 54, 661

Tully R. B., Libeskind N. I., Karachentsev I. D., Karachentseva V. E., Rizzi L., Shaya E. J., 2015, ApJ, 802, L25

Vegetti S., Koopmans L. V. E., Auger M. W., Treu T., Bolton A. S., 2014, MNRAS, 442, 2017

Walker M. A., 1999, MNRAS, 308, 551

Walt S. v. d., Colbert S. C., Varoquaux G., 2011, Computing in Science \& Engineering, 13, 22

Wang L., Dutton A. A., Stinson G. S., Macciò A. V., Gutcke T., Kang X., 2016, preprint, (arXiv: 1601.00967 )

Weinberg D. H., Bullock J. S., Governato F., Kuzio de Naray R., Peter A. H. G., 2015, Proceedings of the National Academy of Science, 112, 12249

Weinmann S. M., van den Bosch F. C., Yang X., Mo H. J., Croton D. J., Moore B., 2006, MNRAS, 372, 1161

Westmeier T., Staveley-Smith L., Calabretta M., Jurek R., Koribalski B. S., Meyer M., Popping A., Wong O. I., 2015, MNRAS, 453, 338

Wilkinson M. I., Evans N. W., 1999, MNRAS, 310, 645

Wojtak R., Mamon G. A., 2013, MNRAS, 428, 2407

Wolf J., Martinez G. D., Bullock J. S., Kaplinghat M., Geha M., Muñoz R. R., Simon J. D., Avedo F. F., 2010, MNRAS, 406, 1220

Wu H.-Y., Hahn O., Wechsler R. H., Behroozi P. S., Mao Y.-Y., 2013, ApJ, 767,23

Zhu G., Zheng Z., Lin W. P., Jing Y. P., Kang X., Gao L., 2006, ApJ, 639, L5 


\section{E. Garaldi et al.}

Zhu Q., Marinacci F., Maji M., Li Y., Springel V., Hernquist L., 2016, MN-

RAS, 458,1559

This paper has been typeset from a $\mathrm{T}_{\mathrm{E}} \mathrm{X} / \mathrm{LT}_{\mathrm{E}} \mathrm{X}$ file prepared by the author. 